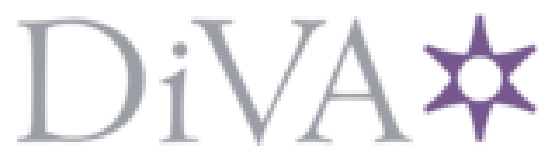

http://www.diva-portal.org

This is the published version of a paper published in J. Chem. Phys.

Citation for the original published paper (version of record):

The following article appeared in:

Johari,G.P., Andersson, O. (2015)

Effects of stacking disorder on thermal conductivity of cubic ice

J. Chem. Phys., 143, 054505 (2015).

and may be found at http://dx.doi.org/10.1063/1.4927566

Access to the published version may require subscription. N.B. When

citing this work, cite the original published paper.

Copyright 2015 American Institute of Physics. This article may be downloaded for personal use only. Any other use requires prior permission of the author and the American Institute of Physics.

Permanent link to this version:

http://urn.kb.se/resolve?urn=urn:nbn:se:umu:diva-108140 


\title{
Effects of stacking disorder on thermal conductivity of cubic ice
}

\author{
G. P. Johari ${ }^{1, a)}$ and Ove Andersson ${ }^{2}$ \\ ${ }^{1}$ Department of Materials Science and Engineering, McMaster University, Hamilton, Ontario L8S 4L7, Canada \\ ${ }^{2}$ Department of Physics, Umeå University, 90187 Umeå, Sweden
}

(Received 11 May 2015; accepted 17 July 2015; published online 4 August 2015)

\begin{abstract}
Cubic ice is said to have stacking disorder when the $\mathrm{H}_{2} \mathrm{O}$ sequences in its structure (space group $F d \overline{3} m$ ) are interlaced with hexagonal ice (space group $P 6_{3} / m m c$ ) sequences, known as stacking faults. Diffraction methods have shown that the extent of this disorder varies in samples made by different methods, thermal history, and the temperature $T$, but other physical properties of cubic and hexagonal ices barely differ. We had found that at $160 \mathrm{~K}$, the thermal conductivity, $\kappa$, of cubic ice is $\sim 20 \%$ less than that of hexagonal ice, and this difference varies for cubic ice samples prepared by different methods and/or subjected to different thermal history. After reviewing the methods of forming cubic ice, we report an investigation of the effects of stacking disorder and other features by using new data, and by analyzing our previous data on the dependence of $\kappa$ on $T$ and on the pressure. We conclude that the lower $\kappa$ of cubic ice and its weaker $T$-dependence is due mainly to stacking disorder and small crystal sizes. On in situ heating at 20-50 MPa pressure, $\kappa$ increases and cubic ice irreversibly transforms more sharply to ice $\mathrm{Ih}$, and at a higher $T$ of $\sim 220 \mathrm{~K}$, than it does in ex situ studies. Cooling and heating between 115 and $130 \mathrm{~K}$ at $0.1 \mathrm{~K} \mathrm{~min}^{-1}$ rate yield the same $\kappa$ value, indicating that the state of cubic ice in these conditions does not change with time and $T$. The increase in $\kappa$ of cubic ice observed on heat-annealing before its conversion to hexagonal ice is attributed to the loss of stacking faults and other types of disorders, and to grain growth. After discussing the consequences of our findings on other properties, we suggest that detailed studies of variation of a given property of cubic ice with the fraction of stacking faults in its structure may reveal more about the effect of this disorder. A similar disorder may occur in the mono-layers of $\mathrm{H}_{2} \mathrm{O}$ adsorbed on a substrate, in bulk materials comprised of two dimensional layers, in diamond and in Zirconium and in numerous other crystals. @ 2015 AIP Publishing LLC. [http://dx.doi.org/10.1063/1.4927566]
\end{abstract}

\section{INTRODUCTION}

States of water frozen at ambient or lower pressures have an obvious role in the occurrence of natural and biological phenomena in different regions of the Earth and its atmosphere. Under most conditions, water freezes to hexagonal ice, ice Ih (space group $P 6_{3} / m m c$ ), but under certain laboratory conditions it can freeze to produce cubic ice (space group $F d \overline{3} m) .{ }^{1-5}$ In nature, freezing of supercooled water droplets is known to produce crystals of cubic ice in cirrus clouds ${ }^{6-8}$ and formation of small crystals of cubic ice in the troposphere and the stratosphere produces certain less frequently observed natural phenomena. ${ }^{9,10}$ Cubic ice, which is mechanically isotropic, also tends to form smaller crystals in comparison with ice $\mathrm{Ih}$, and therefore its formation is considered to prevent freeze-damage to natural and synthetic materials in industrial processes that require cooling to low temperatures.

Historically, papers reporting that a new phase of ice forms on cooling water or its vapors at ambient pressure have appeared since $1896 .{ }^{11-14}$ In 1943, König ${ }^{15}$ determined the crystal structure by using X-ray diffraction from an ice made by water vapor-deposition on a cold substrate, and he found that the diffraction pattern of the deposited ice belongs

\footnotetext{
a)Electronic mail: joharig@mcmaster.ca
}

to cubic-symmetry. He named the vapor deposited ice as cubic ice. ${ }^{15}$ Whalley and coworkers later made cubic ice by recovering the high pressure crystalline phases of ices, namely, ice II, ${ }^{16-18}$ ice III, ${ }^{16,17,19}$ and ice $\mathrm{V}^{16,17}$ at 1 bar pressure and $\sim 77 \mathrm{~K}$, and then heating it ex situ, at 1 bar pressure, i.e., outside their conditions of stability. They named it ice Ic. ${ }^{16,17}$ Ice Ic has been made by vapor-deposition on a substrate kept usually at $77 \mathrm{~K}^{15,20,21}$ and it has also been made by heating the amorphous solid water that was formed by vapor-deposition. ${ }^{21}$ In 1984, it was found that ice Ic forms also when the high-density amorph, HDA, produced by collapsing ice $\mathrm{Ih}^{22}$ or ice $\mathrm{Ic}^{23}$ at $\sim 1 \mathrm{GPa}$ pressure was heated at pressures at $0.1-50 \mathrm{MPa}$ range. The heating transformed HDA to the lowest density amorph, LDA, which then transformed to ice Ic. In 1987, it was reported that ice Ic forms when aqueous solutions of $\mathrm{LiCl}^{24}$ and of glycerol ${ }^{25}$ were cooled or cold-crystallized on heating, and when 3-5 $\mu \mathrm{m}$ size droplets of water in an aerosol were deposited on a copper plate kept at $\sim 190 \mathrm{~K} .{ }^{1}$ The latter procedure produced glassy water when the substrate was kept at $\sim 77 \mathrm{~K} .^{2}$ This glassy water became ultraviscous liquid when heated to a temperature above $148 \mathrm{~K}$, which then crystallized to ice Ic. $^{2}$ Later studies ${ }^{26}$ showed that LDA also became ultraviscous liquid before crystallizing to ice Ic. Methods used to produce ice Ic until the year 2005 were described in the context of interfacial energy and enthalpy, and the conditions of ice Ic-ice Ih phase inversion. ${ }^{27}$ 
Since 2005, a variety of other methods have produced ice Ic. These are: (a) cooling at $10-30 \mathrm{~K} \mathrm{~min}^{-1}$ of smaller than $0.9 \mu \mathrm{m}$ size droplets dispersed in emulsions (water, lanolin, and mineral oil) to a temperature $T$ below $240 \mathrm{~K},{ }^{8,28}$ and (b) decomposing ice clathrates. ${ }^{29-31}$ Kuhs et al. ${ }^{31}$ provided a chart of the different routes by which ice Ic has been made (Fig. 2, Ref. 31), (i) by vapor deposition, (ii) by warming of amorphous ices, (iii) by freezing of confined water, of emulsified water, gels, aqueous solutions of electrolytes and nonelectrolytes, and hyperquenching of $\mu \mathrm{m}$-size water droplets, (iv) by ex-situ warming of high pressure ices, II, III or IX, IV, ${ }^{32}$ $\mathrm{V}$, and $\mathrm{VIII}^{33}$ and $\mathrm{XII},{ }^{34}$ recovered at ambient pressure, and, (v) by decomposition of $\mathrm{CO}_{2}$ and $\mathrm{CH}_{4}$ gas clathrate hydrates. ${ }^{35}$ (See also Table I, Ref. 36 for a summary of the diffraction data of cubic and hexagonal ices formed by different methods.) In a discussion of the transformations of the so-called ice Ic, Kuhs et al. wrote: ${ }^{35}$ "It was noticed early on ${ }^{14}$ that the diffraction patterns for ice Ic obtained from different starting materials were different. These differences were explained by Kuhs et al. ${ }^{15}$ in terms of various degrees of stacking faulting for ice Ic from different origins. The faults were identified as deformation stacking faults, which in diffraction experiments lead to the appearance of broad reflections at Bragg angles typical for ice Ih as well as to high- and/or low-angle shoulders on the Bragg peaks at genuine ice Ic positions. ${ }^{15}$ The width of the cubic reflections was used to estimate the particle size of ice Ic produced from ice II as $160 \AA$. Stacking faults in ice Ih and their creation by rapid temperature changes were also described by other authors." ${ }^{16}$ (References 14-16 in the quoted text are here Refs. 18,37 , and 45, respectively, the last being the cit. to Lattice Defects in ice Crystals, X-ray Topographic observations.)

Kuhs et $a l^{37}$ found several unexpected features in the neutron diffraction spectra of ice Ic made by heating ice II at 1 bar pressure: (i) There was a small peak at the position where the (100) reflection peak of ice Ih is found, ${ }^{38,39}$ (ii) the (111) reflection peak of cubic ice was unusually broadened, and (iii) there was a significant difference between the relative intensities of the observed peaks from those calculated for the ideal structures of ice Ih (space group $P 6_{3} / \mathrm{mmc}$ ) and of ice Ic (space group $F d \overline{3} m$ ). ${ }^{38-40}$ (The unit cells of ices Ih and Ic are illustrated in Fig. 1 inset here.) After providing comprehensive details of the previous studies, Kuhs et al. ${ }^{37}$ concluded that ice Ic contains congruent, interlaced sequences of ice Ih, like stacking faults. The three diffraction features attributed to stacking disorder in cubic ice were found to be only qualitatively similar among the samples of the cubic ices that were made by vapor-deposition, by crystallizing emulsified water droplets, and by decomposing ice clathrates by heating at different pressures, ${ }^{35}$ and some high pressure ices by heating at ambient pressure. To state precisely, they differ in details of the relative intensities and broadening of the diffraction peaks. Some of these differences arise from the stacking disorder introduced by their preparation method and some probably from thermal or mechanical treatment of the ice samples after they were made.

It should be pointed out that occurrence of stacking faults has been known as general phenomena in the studies of crystal structures and physical properties of metals. ${ }^{41-43}$ In 1952, Paterson $^{43}$ had described these faults as follows: "When a

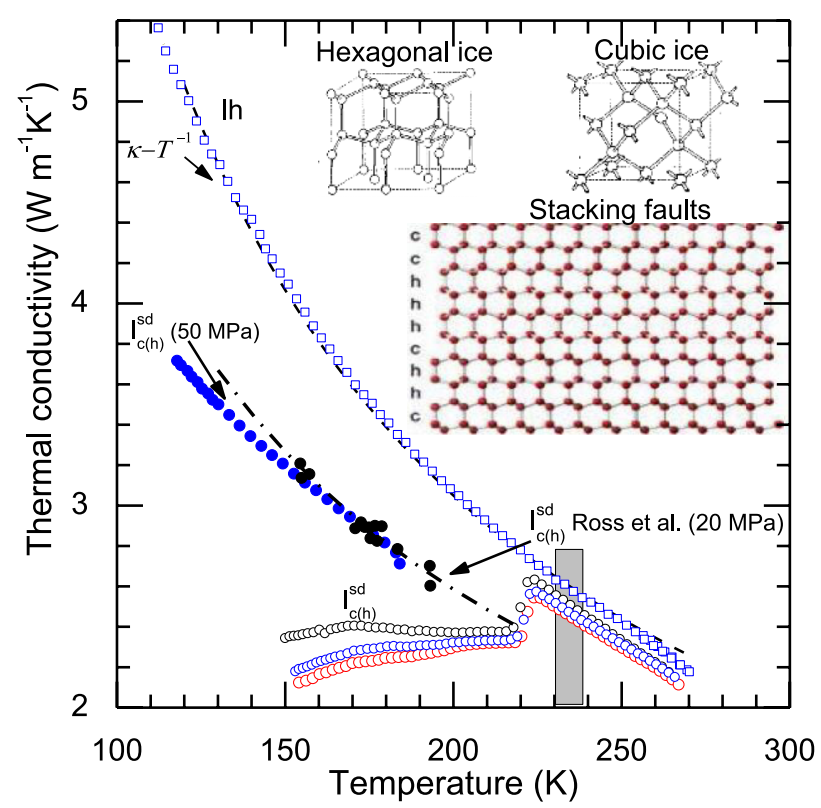

FIG. 1. The plots of $\kappa$ of ice $\mathrm{I}_{\mathrm{c}(\mathrm{h})}^{\mathrm{sd}}$ and ice Ih against $T$. New data for ice $\mathrm{I}_{\mathrm{c}(\mathrm{h})}^{\mathrm{sd}}$ formed by heating ice II to $185 \mathrm{~K}$ at $50 \mathrm{MPa}$, subsequent cooling and temperature cycling in the $115-130 \mathrm{~K}$ range (blue dots). Data for ice $\mathrm{I}_{\mathrm{c}(\mathrm{h})}^{\mathrm{sd}}$ measured on cooling from $200 \mathrm{~K}$ at $20 \mathrm{MPa}$, after the state had been obtained by heating ice II to $200 \mathrm{~K}$ from Ref. 19 (black dots). Ice $\mathrm{I}_{\mathrm{c}(\mathrm{h})}^{\mathrm{sd}}$ formed by heating of HDA at $40 \mathrm{MPa}$ (red circles), at $50 \mathrm{MPa}$ (blue circles), and at $70 \mathrm{MPa}$ (black circles). ${ }^{69,70}$ These samples of ice $\mathrm{I}_{\mathrm{c}(\mathrm{h})}^{\mathrm{sd}}$ transform to ice Ih at $220.0 \mathrm{~K}, 219.4 \mathrm{~K}$, and $218.1 \mathrm{~K}$, respectively. The samples were prepared by freezing a new sample of water and forming HDA by pressure-amorphization at $T \sim 130 \mathrm{~K}$, reducing the pressure to $40 \mathrm{MPa}, 50 \mathrm{MPa}$, or $70 \mathrm{MPa}$ simultaneously as the temperature was lowered, and then heating the HDA from a temperature in the $80-120 \mathrm{~K}$ range to $150 \mathrm{~K}$. Data for ice Ih are for the sample formed by freezing water at $0.1 \mathrm{MPa}$ and thereafter cooling simultaneously as the pressure was slowly raised to $70 \mathrm{MPa}$ in the $260-230 \mathrm{~K}$ range (blue squares). The dashed line is for the shape of a plot when $\kappa$ varies as $1 / T$, and the dashed-dotted line shows the equation for $\kappa$ suggested for ice $I_{c(h)}^{s d}(150 \mathrm{~K}-200 \mathrm{~K})^{19}$ in an extended range $130-220 \mathrm{~K}$. The gray field indicates the range when pure water emulsions freeze partly to ice $\mathrm{I}_{\mathrm{c}(\mathrm{h})}^{\mathrm{sd}}$ at $10-30 \mathrm{~K} \mathrm{~min}^{-1}$ cooling rates. ${ }^{8,28,36}$ The insets show the unit cells of ice Ih and ice Ic, and an illustration of stacking faults (c refers to cubic and $\mathrm{h}$ to hexagonal; after modification from Ref. 59). The unit cell dimensions of ice Ih and ice Ic are on different scales. For ice Ih, $a=0.448 \mathrm{~nm}$ and $c=0.731 \mathrm{~nm}$. For ice Ic, $a=0.637 \mathrm{~nm}$. (The illustration for stacking faults was adapted with permission from Carr et al., J. Phys. Chem. Lett. 5, 2469 (2014). Copyright 2014 American Chemical Society.)

crystalline lattice is conceived in terms of the stacking of sequences in a well-defined manner, any error in the regular sequence of the sequences is known as a stacking "fault," "mistake," or "disorder." From x-ray diffraction studies, it is known that stacking faults may arise in the growth of crystals with well-developed sequence structures, for example, micas, ${ }^{1,2}$ graphite, ${ }^{3}$ silicon carbide, ${ }^{3}$ uranyl chloride, ${ }^{4}$ and others. Prominent faulting can also arise during martensitic phase transformations, for example, in cobalt ${ }^{5}$ and lithium.." (References in the quoted text are in the original.) Paterson ${ }^{43}$ also described how the stacking disorder can be quantified by analysis of the X-ray diffraction data.

Returning to the stacking sequences in the ices, the facecentered cubic lattice of ideal ice Ic is $A B C A B C A B C$ and the stacking sequence in the open hexagonal lattice of ideal ice Ih is $\mathrm{ABABAB}$. Randomly dispersed $\mathrm{ABABAB}$ sequence (sequences) of ice Ih in the structure of ice Ic is known as 
stacking faults. (See inset in Fig. 1.) Hondoh et al. ${ }^{44}$ studied the formation and annihilation of stacking faults in fresh ice Ih crystals grown from high purity water and they estimated the stacking fault energy as $0.31 \mathrm{~mJ} \mathrm{~m}^{-2}\left(3 \times 10^{-4} \mathrm{eV}\right.$ per molecule in the plane). ${ }^{45}$ Petrenko and Whitworth ${ }^{40}$ comprehensively discussed the formation of stacking faults by glide dislocations, and they described the related detailed work on the stacking faults by the Sapporo group..$^{45}$ We refer the reader to pp. 176-180 in Ref. 40 for description of the structure of stacking faults in ice and the methods of their observations, which include X-ray topography, the method used to study stacking faults formed by deformation of ice Ih. Kuhs et al. ${ }^{37}$ studies of vapor-deposited ice Ic by neutron scattering and their conclusive arguments for the existence of stacking faults in ice Ic provided an entry point to almost all studies of the stacking disorder in ice I.

Murray and Bertram ${ }^{8}$ found that when emulsions containing $\mu \mathrm{m}$ size droplets of water were formed by using a mixture of $10 \%$ surfactant (lanolin) in paraffin oil and the emulsion cooled to $T<232 \mathrm{~K}$, the droplets of size less than $0.9 \mu \mathrm{m}$ in the emulsion froze to ice Ic and droplets of size larger than $\sim 0.9 \mu \mathrm{m}$ size froze to ice $\mathrm{Ih}$. The X-ray diffraction features of their ice Ic corresponded to the neutron diffraction features of the ice Ic found by Kuhs et al. ${ }^{37}$ who, 18 yr earlier, had interpreted their findings ${ }^{37}$ in terms of the stacking faults. In particular, the X-ray and neutron diffraction features were similar in terms of the appearance of the (100) diffraction peak and symmetric and asymmetric broadening of the cubic ice peaks, prepared by other methods. ${ }^{35}$ (See Fig. 3 and Table I in Ref. 36.) The above-mentioned similarity between the diffraction features led to the conclusion that the ice Ic which Murray and coworkers made $e^{8,28,36}$ also contained stacking faults. Since Murray and Bertram's ${ }^{8}$ finding in 2006, ice Ic has been intensively studied by experiments and by computer simulation. ${ }^{46-49}$ In a perspective on the stacking disorder in ice Ic with extensive citations, first Kuhs et al. ${ }^{31}$ and then Malkin et $a .^{36}$ stressed that the ice Ic samples made by different methods show only qualitatively similar diffraction features; there are significant differences in details, as described above. These differences occur in the relative heights of the diffraction peaks and their broadness in samples prepared by different methods and studied at ambient or sub-ambient pressures. The differences appear to be related to the parent phase.

The feature attributed to stacking faults in Refs. 31 and 36 was obvious in the high-resolution data in Ref. 31. It was, however, absent in the (perhaps low-resolution) X-ray diffraction data of ice Ic formed by heating HDA in the study by Bosio et al. ${ }^{50}$ The need for good quality diffraction data in such a study was later stressed by Kuhs et al., ${ }^{31}$ who wrote, "The unambiguous assignment of the degree of stacking disorder requires good-quality diffraction data. Differences with respect to ice Ih are small, in the range from 200 to $240 \mathrm{~K}$, and easily unnoticed in lower-resolution, lower-quality data." Alternatively, one may suggest that in the study of Bosio et al., ${ }^{50}$ heating of HDA produced the least hexagonal stackings as it transformed to ice Ic via LDA. Nevertheless, it seems prudent that before accepting the proposed ${ }^{31,36}$ non-existence of real (stacking-fault free) ice Ic, we try to understand why and how stacking faults are produced not only in the structure of ice Ic but also in the structure of other materials, ${ }^{43}$ and what are the energetic consequences of these faults.

The diffraction feature of ice Ic that could be attributed exclusively to stacking faults is the (non-cubic) ice Ih (100) reflection peak at $d$-spacing of $3.86 \AA,{ }^{37}$ the so-called stacking fault peak. They suggested ${ }^{31,36}$ that previous studies were most likely performed not on ice Ic, but on a structure containing stacking faults, and therefore the solid known as ice Ic is a stacking-disordered structure; ideal ice Ic may not exist. They noted, ${ }^{36}$ (i) that the halo at $\sim 28^{\circ}$ from the Sun, observed in 1630 by Scheiner, and explained by Whalley, ${ }^{9,10}$ and which has been more recently photographed, ${ }^{51}$ can be explained only by the presence of octahedral crystals of ice I, ${ }^{9,10,52}$ (ii) electron micrographs have shown that a crystal of cubic symmetry lies at the center of a snow flake, ${ }^{53,54}$ (iii) crystals of four-fold symmetry have been occasionally found to appear in the polar stratosphere, ${ }^{55}$ and (iv) ice formed by vapor deposition at $T$ of $\sim 200 \mathrm{~K}$ has an apparent cubic morphology ${ }^{56}$ (cit. 141-144 in Ref. 8). Since a study that first gave the structure of HDA did not find the $3.86 \AA$ peak in a well-annealed ice Ic formed by heating HDA, ${ }^{50}$ it is possible that ideal ice Ic, i.e., without stacking faults, may exist.

It is known that crystallographic densities of ice Ic and Ih are identical, but the real densities are expected to differ by an amount that depends upon the difference between the type and population of the crystal defects present in them. Ice Ih is also mechanically anisotropic, and the crystal growth patterns of the two differ. There are also other small differences: (i) Both the infrared ${ }^{58}$ and the Raman spectra ${ }^{59}$ of ice Ic differ from those of ice Ih. (ii) The enthalpy of ice Ic is higher than that of ice Ih. ${ }^{60-63}$ (Ice Ic transforms to ice Ih.) (iii) The vapor pressure of ice Ic is $10.5 \%$ higher than that of ice $\mathrm{Ih}$ in 180-190 K range. ${ }^{64}$ (Note that smaller size particles have a higher vapor pressure than larger size particles. So, the higher vapor pressure of ice Ic relative to ice Ih can also be partly due to the smaller size of the ice Ic crystals.) Some of these differences are intuitively expected because the structure and density generally determine a crystal allotrope's properties, and the O-O-O angles in ice Ic are tetrahedral $\left(109.5^{\circ}\right)$, but the O-O-O angles in ice Ih deviate from the perfect tetrahedral value, ${ }^{65,66}$ thereby causing the $\mathrm{H}-\mathrm{O}-\mathrm{H}$ (hydrogen bonds) in ice Ih to become slightly nonlinear. It is also known that the structure of ice Ih contains channels along the c-axis. ${ }^{38-40}$ As early as in 1984, Kiefte et al. ${ }^{52}$ had noted that "Ice Ic always nucleates in preference to ice Ih from a metastable phase of water below $\sim 200 \mathrm{~K}$, and sometimes nucleates from liquid at $\sim 235$ K."

Our interest in the subject arose on reading the perspectives of Kuhs et al. ${ }^{31}$ and of Malkin et al. ${ }^{36}$ on the stacking disorder in ice Ic, and the report of Hansen et al. ${ }^{57}$ showing that the rate of irreversible conversion of ice Ic to ice Ih under isothermal conditions at 1 bar pressure is higher at higher $T$. (See also Fig. 3 in Ref. 31.) They noted that properties of ice Ic barely differ from those of ice Ih. In previous studies, we had found that a phonon scattering property, namely, the thermal conductivity, $\kappa$, of ice Ic is $\sim 20 \%$ less than that of ice Ih at $\sim 160 \mathrm{~K},,^{19,67-70}$ which is much more than the difference in their other properties. Here, we provide data from a study of $\kappa$ of ice Ic under pressure and analyze the new data and some of 
our previously published data. Hence, we investigate, (i) the effects of stacking disorder on $\kappa$ of ice Ic, and (ii) the effects of the parent phase, the production method, and annealing of stacking disordered ice Ic on its thermal conductivity. The findings are significant for understanding the effects of this kind of disorder on both the properties of ice, and the occurrence of certain natural phenomena.

Two nomenclatures have been suggested for stacking disordered ice I. Malkin et al. ${ }^{36}$ suggested using the abbreviation, ice $\mathrm{I}_{\mathrm{sd}}$. This term included samples of ice Ic containing varying amounts of ice Ih sequences, as well as samples of ice Ih containing varying amounts of ice Ic sequences. In their latest review, Hansen et al..$^{71}$ extended the diffraction data analysis performed by Malkin et al. ${ }^{36}$ On the basis of the extended analysis, they ${ }^{71}$ suggested considering another abbreviation that may be seen as a continuum from ideal ice Ic to ideal ice Ih through a range over which the amount of stacking sequences continuously vary. These sequences are initially those of ice Ih in ice $\mathrm{Ic}^{31,35-37}$ and finally those of ice Ic in ice Ih. ${ }^{71}$ Ice Ic containing hexagonal sequences is ice $\mathrm{I}_{\mathrm{c}(\mathrm{h})}$, and ice Ih containing cubic sequences is $\mathrm{I}_{(\mathrm{c}) \mathrm{h}}$. (The stacking fault sequence is in parenthesis.) Hence, they suggested ${ }^{71}$ a transformation sequence: $\mathrm{I}_{\mathrm{c}(\mathrm{h})} \rightarrow \mathrm{I}_{\mathrm{ch}} \rightarrow \mathrm{I}_{(\mathrm{c}) \mathrm{h}} \rightarrow \mathrm{Ih}$. (They excluded ice Ic from this sequence because there is no clear experimental evidence for the existence of cubic ice without stacking faults, and hence this phase, previously known as ice Ic, became a "hypothetical" entity. ${ }^{31}$ ) They clearly noted that there is not one solid, but numerous solids named ice I. Specifically, there is a range of cubic ices containing varying amounts of ice Ih sequences and there is a range of hexagonal ices containing varying amount of ice Ic sequences. (See cit. to Bartel-Rausch et al. in Ref. 37 here.) In all cases, loss of ice Ic sequences in a sample may be a written as ice $I_{c \rightarrow h}$ conversion. Kuhs et al. noted: ${ }^{31}$ "What has been called "cubic ice" for $70 \mathrm{yr}$ now turns out to be arguably the most faceted ice phase in a literal and more general sense." Despite the admitted variability of sequences of one type of ice in the structure of the other type, it has been suggested that stacking disordered ice Ic be regarded as a distinct phase of ice in the trigonal crystal system, ${ }^{31}$ a subgroup of the hexagonal crystal family, previously recognized by Hallet $e t$ al. ${ }^{7}$

One expects that as a distinct phase, all samples of the stacking disordered ice I, regardless of the method of their preparation, thermal history, particle size, and temperature and pressure, would show not only the presence of a diffraction peak at $d$-spacing of $3.86 \AA$, but also the same ratio of intensity and of width of the $d$-spacing peak to the intensity and the width of all other diffraction peaks. If that is not found, then one may need to show that the differences in the details of diffraction features are due to varying amounts of the point defects, interfaces, and dislocations in the samples. Until that is done, we suggest that the nomenclature suggested by Hansen et al. ${ }^{71}$ and by Malkin et al. ${ }^{36}$ be maintained. For convenience, we use the subscripts as suggested in Ref. 71 and indicate the stacking disorder by the superscript "sd." (In Ref. 36, it is used as a subscript.) Hence, we use the combination of their terms, ice Ic, ice $\mathrm{I}_{\mathrm{c}(\mathrm{h})}^{\mathrm{sd}}$, ice $\mathrm{I}_{\mathrm{ch}}^{\mathrm{sd}}$ ice $\mathrm{I}_{(\mathrm{c}) \mathrm{h}}^{\mathrm{sd}}$ ice $\mathrm{Ih}$, to indicate that ice Ic may still exist. In the cases of ice $\mathrm{I}_{\mathrm{ch}}^{\mathrm{sd}}$, ice $\mathrm{I}_{\mathrm{ch}}^{\mathrm{sd}}$, and ice $\mathrm{I}_{(\mathrm{c}) \mathrm{h}}^{\mathrm{sd}}$, there would be characteristic diffraction peaks of both ice Ic and Ih sequences, but their relative intensities and width would vary. Thus, in the terminology of Hansen et al. ${ }^{71}$ the transformation sequence is: ice Ic $\rightarrow$ ice $\mathrm{I}_{\mathrm{ch})}^{\mathrm{sd}} \rightarrow$ ice $\mathrm{I}_{\mathrm{ch}}^{\mathrm{sd}} \rightarrow$ ice $\mathrm{I}_{(\mathrm{c}) \mathrm{h}}^{\mathrm{sd}} \rightarrow$ ice Ih. This sequence would serve the purpose of treating stacking faults as a nanoscopic disorder of layered structure in a single crystal, and we use it here.

\section{EXPERIMENTAL METHODS AND RESULTS}

In previous studies, we used thermal conductivity as a characterizing feature of the ice polymorphs. These included studies of (i) the structural transformation of the ices under pressure, ${ }^{67}$ (ii) the proton-ordering in the structure of ice $\mathrm{Ih},{ }^{68}$ (iii) the pressure-induced amorphization by structural collapse of ice $\mathrm{Ih}^{69,70}$ and of Ic, ${ }^{70}$ and, (iv) the transformation of the collapsed structures to LDA on heating. ${ }^{69}$ The procedure for determining $\kappa$ under varying conditions of pressure $p$ and $T$ has been described previously. ${ }^{67}$ Briefly, we used a hot wire method in a sample of diameter $38 \mathrm{~mm}$ and depth $15 \mathrm{~mm}$ contained in a Teflon cell. There was a need to keep the solid sample under slight pressure to maintain physical contact between the solid and the hot wire while measuring its $K$ as a function of the temperature $T$. Therefore, our data were obtained by keeping the sample and the wire under a pressure of higher than $40 \mathrm{MPa}$, and this pressure was corrected for the friction between the pressure assembly and the sample. Thus, we investigated the effects of both $T$ and $p$ on the $\kappa$ value without removing the ice $\mathrm{I}_{\mathrm{c}(\mathrm{h})}^{\mathrm{sd}}$ or ice $\mathrm{Ih}$ samples from the high pressure vessel in which they were made. The particle size in the samples is not known, but the samples had no voids.

To investigate the effects of the parent or precursor phase, we made the ice $I_{c(h)}^{\text {sd }}$ samples by two methods, (i) by heating ice II under pressure and, (ii) by heating HDA under pressure. Previous studies of $\kappa$ of ice Ih and ice $I_{c(h)}^{\text {sd }}{ }^{19,67-70}$ formed by heating ice II, ${ }^{19,70} \mathrm{HDA}$, and $\mathrm{LDA}^{69,70}$ had shown that $\kappa$ of ice $I_{c(h)}^{\text {sd }}$ depends upon thermal history but remains less than that of ice Ih, and its temperature-dependence is weaker than that of ice Ih. As was the case in some previous studies of ice $\mathrm{I}_{\mathrm{c}(\mathrm{h})}^{\mathrm{sd}}, 58,59$ we did not characterize the ice $\mathrm{I}_{\mathrm{c}(\mathrm{h})}^{\mathrm{sd}}$ or ice $\mathrm{Ih}$ samples by X-ray or neutron diffraction methods. Instead, we identified the various ices by methods of their preparation, their $\kappa$ values, and the variation of their $\kappa$ with $T$.

The plots of $\kappa$ of ice Ih and of ice $\mathrm{I}_{\mathrm{c}(\mathrm{h})}^{\mathrm{sd}}$ against $T$ are shown in Fig. 1, where the samples are identified by the method of their preparation. For comparison, the $T$-range over which ice $\mathrm{I}_{\mathrm{c}(\mathrm{h})}^{\mathrm{sd}}$ formed by freezing emulsified water, ${ }^{8,28}$ at $0.1 \mathrm{MPa}$ and $T<232 \mathrm{~K}$ is indicated. The plots of $\kappa$ of ice $\mathrm{I}_{\mathrm{c}(\mathrm{h})}^{\mathrm{sd}}$ against the pressure in the $50 \mathrm{MPa}-150 \mathrm{MPa}$ range measured at $129 \mathrm{~K}$ are shown in Fig. 2, where the plots of $\kappa$ of ice Ih measured at $130 \mathrm{~K}$ over the $120 \mathrm{MPa}-150 \mathrm{MPa}$ pressure range are included. The fitted line indicates values obtained by extrapolation to lower pressures.

Hansen et $a l^{57}$ and Malkin et $a .^{36}$ used the term "cubicity" as a quantitative measure of stacking disorder determined by fitting the X-ray and neutron diffraction features. We use a simpler term, namely, the total number of ice Ih sequences, $n_{\mathrm{Ih}, \text { seq }}$, considered as stacking faults interlaced in the total number of ice Ic sequences $n_{\text {Ic, seq }}$, without considering the sequence in which each of the two types of sequences is 


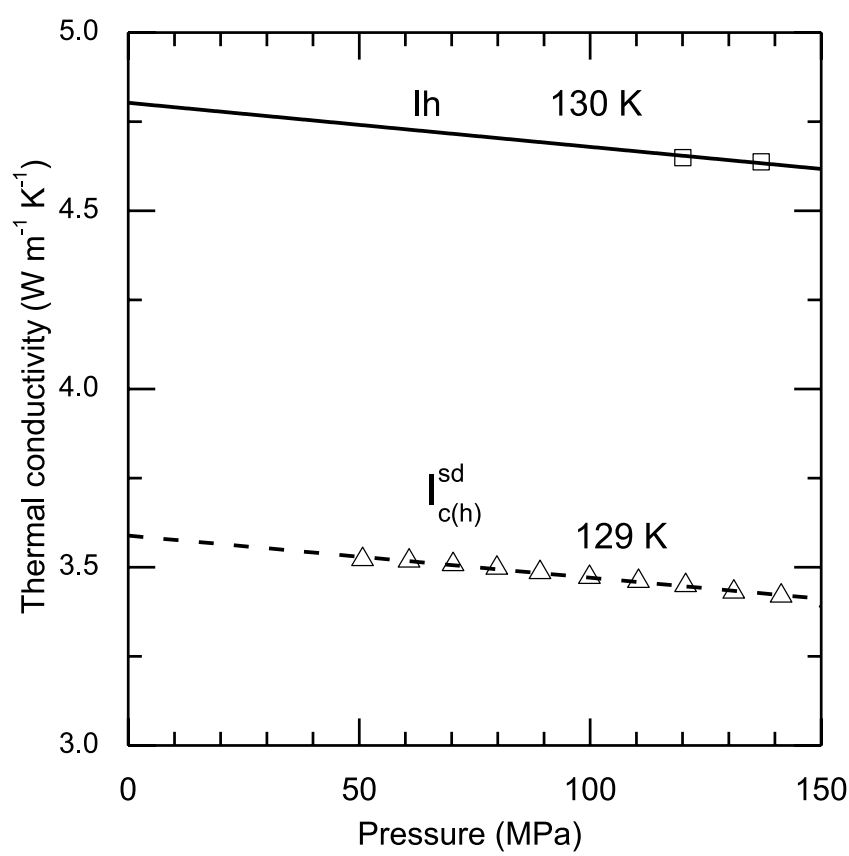

FIG. 2. Thermal conductivity plotted against pressure for ice $I_{h}$ and ice $\mathrm{I}_{\mathrm{c}(\mathrm{h})}^{\mathrm{sd}}{ }^{69,70}$

interlaced. So, the ratio $\chi=\left[n_{\mathrm{Ic}, \mathrm{seq}} /\left(n_{\mathrm{Ic}, \mathrm{seq}}+n_{\mathrm{Ih}, \mathrm{seq}}\right)\right]$, which we use for clarity. ( $\chi=0.44$ in inset of Fig. $1, \chi<0.5$ when $n_{\mathrm{Ic}, \text { seq }}<n_{\mathrm{Ih}, \mathrm{seq}}, \chi=0.5$ when $n_{\mathrm{Ic}, \text { seq }}=n_{\mathrm{Ih}, \mathrm{seq}}$, and $\chi>0.5$, when $n_{\mathrm{Ic}, \text { seq }}>n_{\mathrm{Ih} \text {, seq }}$, i.e., stacking faults occur in ice Ic.) This ratio corresponds to cubicity, $\phi_{c}$, the term first used by Hansen et al. ${ }^{57}$ and discussed in detail by Malkin et al. ${ }^{28}$ (One has to also maintain the possibility that, because of the limited accuracy of the data and analysis, the current diffraction methods are unable to detect hexagonal sequences in ice Ic and cubic sequences in ice Ih below a certain value.) In this context, we found Paterson's conclusion instructive: ${ }^{43}$ "However, from the microstructure of copper-silicon alloys $\left(\right.$ Barrett $\left.{ }^{1}\right)$ it seems likely that the faults are not distributed at random but are clustered on a microscopic scale. In this case, the expressions for the intensity distribution of the broadening will not be strictly true, although qualitatively the behavior should be similar. When extreme clustering occurs, the x-ray effects can be thought of as the superposition of those for heavy-faulting and for very slight or no faulting." Computer simulations (cit. in Ref. 36) showed that clustering of fault sequences occurs in ice $\mathrm{I}_{\mathrm{ch}}^{\mathrm{sd}}$, which seems consistent with the neutron and X-ray diffraction features. Either cubicity or $\chi$ can be used for the purpose, but we find that the term "cubicity" can be confusing when on one extreme it is used for stacking disorder from hexagonal sequences in ice Ic, as in Refs. 36 and 37 , and on the other extreme, it is used for stacking disorder from cubic sequences in ice Ih.

\section{DISCUSSION}

\section{A. Effects of $p$ and $T$ on stacking faults in ice lc made from ice II and from HDA}

Our studies of cubic ice and ice Ih are distinguished from the previous ones $28,35-37,50,57,71,72$ in several respects. First, we performed in situ measurements on 10-20 $\mathrm{g}$ amount of samples contained in a $38 \mathrm{~mm}$ diameter cylindrical Teflon container kept inside a pressure vessel in which the ices were made by thermal and pressure treatments, while all previous studies of cubic ice were performed ex-situ, as thin films, ${ }^{37}$ as emulsified droplets $^{8,28,36}$ on samples much smaller in mass and size. Second, our samples were formed under pressure and kept under pressure and therefore, they were not porous and their particle size was larger than the $16 \mathrm{~nm}$ to $\mu \mathrm{m}$ size particles in the vapor-deposited, decomposed clathrate hydrates and emulsified droplet samples. Third, we measured $\kappa$ of cubic ice after it had formed by heating ice II at $50 \mathrm{MPa}-185 \mathrm{~K}$ at $0.3 \mathrm{~K} \mathrm{~min}^{-1}$ rate, during the cooling from $185 \mathrm{~K}$ to $115 \mathrm{~K}$ (at initially about the same rate), and thereafter during the heating to $130 \mathrm{~K}$ at $0.1 \mathrm{~K} \mathrm{~min}^{-1}$ rate, while Ross et al. ${ }^{19}$ had made cubic ice by heating ice II from an unspecified low temperature to $200 \mathrm{~K}$ at $20 \mathrm{MPa}$ and measured $\kappa$ of the samples on cooling from $200 \mathrm{~K}$ at $20 \mathrm{MPa}$. Finally, we did a separate study ${ }^{69,70}$ of cubic ice by heating HDA and measured its $\kappa$ only during the heating at pressures of 40,50 , or $70 \mathrm{MPa}$. For these reasons, we refer to the samples we studied as ice $\mathrm{I}_{\mathrm{c}(\mathrm{h})}^{\mathrm{sd}}$. We discuss first the new and the previously published data for ice $\mathrm{I}_{\mathrm{c}(\mathrm{h})}^{\mathrm{sd}}$ formed by heating ice II, and then the previously reported data for ice $\mathrm{I}_{\mathrm{c}(\mathrm{h})}^{\mathrm{sd}}$ formed by heating HDA.

The $\kappa$ against $T$ plots for ice $\mathrm{I}_{\mathrm{c}(\mathrm{h})}^{\mathrm{sd}}$ are shown in Fig. 1. They show that $\kappa$ of ice $\mathrm{I}_{\mathrm{c}(\mathrm{h})}^{\mathrm{sd}}$ changes slowly and irreversibly on heating up to $T$ of $\sim 220 \mathrm{~K}$, and then increases rapidly to the value for ice $\mathrm{Ih}$ at $\sim 220 \mathrm{~K}$. This means that the state of the ice $\mathrm{I}_{\mathrm{c}(\mathrm{h})}^{\mathrm{sd}}$ sample gradually changes on heating irreversibly before (irreversibly) transforming to ice Ih. Kuhs et al. ${ }^{35}$ formed ice $\mathrm{I}_{\mathrm{c}(\mathrm{h})}^{\mathrm{sd}}$ by decomposing $\mathrm{CH}_{4}$ and $\mathrm{CO}_{2}$ clathrate hydrates and studied the kinetics of its slow transformation to ice Ih. They concluded that most stacking faults in the thus formed ice $\mathrm{I}_{\mathrm{c}(\mathrm{h})}^{\mathrm{sd}}$ disappear on heating to $T$ close to $205 \mathrm{~K}$, but some stacking faults persist up to $T$ of $240 \mathrm{~K}$. It is not clear whether the rapid change observed here at $\sim 220 \mathrm{~K}$ is due to the effect of pressure on the transformation or the effect of preparation method of our ice $\mathrm{I}_{\mathrm{c}(\mathrm{h})}^{\mathrm{sd}}$ or both.

The plots in Fig. 2 show that increase in $p$ decreases $k$ of both ice $\mathrm{Ih}^{69}$ and ice $\mathrm{I}_{\mathrm{c}(\mathrm{h})}^{\mathrm{sd}}{ }^{70}$ Therefore, the extrapolated $\kappa$ of ice $\mathrm{I}_{\mathrm{c}(\mathrm{h})}^{\mathrm{sd}}$ at ambient pressure $(0.1 \mathrm{MPa})$ would be slightly higher than the values plotted in Fig. 1.

The recent study of Malkin et al. ${ }^{36}$ showed that $n_{\text {Ic, seq }}$ in ice $\mathrm{I}_{\mathrm{c}(\mathrm{h})}^{\mathrm{sd}}$ formed on heating ice II from $150 \mathrm{~K}$ to $165 \mathrm{~K}$ at ambient pressure depends upon the rate $q$ at which ice II is heated. They wrote: ${ }^{36}$ "There is a monotonic decrease in the fraction of cubic sequences with increasing heating rates, suggesting that increasing the rate of heating promotes the recrystallization of ice II to ice Ih or the transformation of cubic sequences to hexagonal sequences. Thus, cubic stacking seems to be favored kinetically." If a sample of ice II is heated and ice $\mathrm{I}_{\mathrm{c}(\mathrm{h})}^{\mathrm{sd}}$ forms at ambient pressure, increase in $q$ leads to ice $\mathrm{I}_{\mathrm{c}(\mathrm{h})}^{\mathrm{sd}}$ samples with lower $\chi$, or less $n_{\mathrm{Ic} \text {, seq }}$ sequences and more $n_{\mathrm{Ih} \text {,seq. }}$. For the highest $q$ of $30 \mathrm{~K} \mathrm{~min}^{-1}$, they reported (cubicity) $\chi=59 \%$ and for the lowest $0.1 \mathrm{~K} \mathrm{~min}^{-1}$ and $0.5 \mathrm{~K} \mathrm{~min}^{-1} \chi=73 \%$ and $71 \%$, respectively (Table IV, Ref. 36). Thus, for heating rates of less than $0.5 \mathrm{~K} \mathrm{~min}^{-1}$ in our study, $n_{\text {Ic, seq }} \sim 70 \%$ just after recrystallization of ice II, unless 
recrystallization under pressure causes a significant change. After recrystallization of ice II, we find $\kappa \approx 2.6 \mathrm{~W} \mathrm{~m}^{-1} \mathrm{~K}^{-1}$ from data of Ross et al. ${ }^{19}$ at $20 \mathrm{MPa}$ and $175 \mathrm{~K}$, and we observe $\kappa=2.7 \mathrm{~W} \mathrm{~m}^{-1} \mathrm{~K}^{-1}$ at $50 \mathrm{MPa}$ and $180 \mathrm{~K}$. Our sample was thereafter cooled and its $\kappa$ was measured during cooling. On the basis of the previous finding from diffraction studies that the same parent phase gives ice $\mathrm{I}_{\mathrm{c}(\mathrm{h})}^{\mathrm{sd}}$ with similar stacking disorder and similar annealing behavior, our results for the $\kappa$ values on cooling at $50 \mathrm{MPa}$ should correspond to ice $\mathrm{I}_{\mathrm{c}(\mathrm{h})}^{\mathrm{sd}}$ with $n_{\mathrm{Ic}, \mathrm{seq}}=70 \%$, or $70 \%$ cubicity. Nevertheless, this value should be regarded approximate. Ross et al. ${ }^{19}$ heated their sample further to $200 \mathrm{~K}$, which may decrease $n_{\mathrm{Ic} \text {, seq }}$ and $\chi$ but should also increase the crystal sizes, if ice $\mathrm{I}_{\mathrm{c}(\mathrm{h})}^{\mathrm{sd}}$ states formed from ice II changed in a similar manner as the ice $\mathrm{I}_{\mathrm{ch})}^{\mathrm{sd}}$ states produced from ice $\mathrm{V}$ and IX change on heating. ${ }^{71}$ (We further discuss this aspect later in this section.) Despite these microstructural differences, our results agree well with those of Ross et al. ${ }^{19}$ except at the lowest $T$, where the lower $\kappa$ we observed is attributed to the smaller grain size of our ice $\mathrm{I}_{\mathrm{ch}}^{\mathrm{sd}}$.

We now consider the ice $\mathrm{I}_{\mathrm{c}(\mathrm{h})}^{\mathrm{sd}}$ produced by heating HDA. It shows distinctly different values for $\kappa$ from the samples made from ice II, up to $T$ of $220 \mathrm{~K}$. Also, the samples heated at lower $p$ of 40 and $50 \mathrm{MPa}$, which at 1 bar pressure had been found to transform visibly via an amorphous solid (LDA) state, ${ }^{16}$ show lower $K$ values than that heated at higher pressure of $70 \mathrm{MPa}$. The difference between these results must be due to a higher degree of disorder, including stacking disorder, and due to small grain sizes. These states have not been

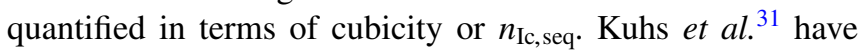
compared the neutron diffraction data of the triplet peak of ice $\mathrm{I}_{\mathrm{c}(\mathrm{h})}^{\mathrm{sd}}$, i.e., the (100), (002), and (101) peaks of ice Ih and (111) peak of ice Ic, measured at $180 \mathrm{~K}$ on the highresolution powder diffractometer $(\lambda \sim 1.594 \AA)$ for samples of ice $I_{c(h)}^{s d}$ prepared by different methods (see Fig. S5 in Ref. 31). These methods were, (i) heating at ambient pressure the recovered samples of ice II, XII, IX, V, and, (ii) heating the recovered LDA that itself was produced by heating HDA. The ice $\mathrm{I}_{\mathrm{c}(\mathrm{h})}^{\mathrm{sd}}$ obtained from ice $\mathrm{V}$ and ice XII showed very similar peak intensities, whereas those of the ice $\mathrm{I}_{\mathrm{c}(\mathrm{h})}^{\mathrm{sd}}$ obtained by other methods differed significantly. In relevance to our study, the neutron diffraction data suggested less stacking faults in ice $\mathrm{I}_{\mathrm{c}(\mathrm{h})}^{\mathrm{sd}}$ derived from LDA via HDA than in ice $\mathrm{I}_{\mathrm{c}(\mathrm{h})}^{\mathrm{sd}}$ obtained from ice II. Thus, if the effects of the high pressures on the population of stacking faults and possibly microstructure (the large crystal sizes and lack of voids in the samples of our study) were insignificant, we could exclude the difference in the population of the stacking faults as the main reason for the difference in $\kappa$. We also note that $\kappa$ of the ice $\mathrm{I}_{\mathrm{c}(\mathrm{s})}^{\mathrm{sd}}$ produced by heating HDA is as much as $20 \%$ less than $\kappa$ of the ice $\mathrm{I}_{\mathrm{c}(\mathrm{h})}^{\mathrm{sd}}$ from ice II at $180 \mathrm{~K}$, and that $\kappa$ remains almost constant on heating. At this stage of these studies, we obtained only qualitative information about the changing microstructure on heating.

Micro-crystalline solids have generally a large contribution to energy from surfaces, known as the grain boundary energy, and deformation adds further energy by adding dislocation in microcrystals. Isothermal or heat annealing of such solids causes grain-growth and thus an irreversible decrease in the energy. Annealing of such solids, therefore, changes the electronic properties, vibrational spectra, mechanical properties, volume, energy, and entropy of the sample. Microstructural details may produce disorder at the crystal interfaces and that impedes phonon propagation, and thus decreases $\kappa$. (Microstructure refers to the crystallite size and the manner in which they are present in a polycrystalline sample, and stacking disorder to the lattice faults in the crystallites.) Among the various properties affected by annealing, the effect on $\kappa$ seems to be largest. For example, $\kappa$ of (mobile) electronfree micro-crystalline solids with other types of structural defects is lower than $\kappa$ of its relatively defect-free single crystal. When it is heated, grain growth on thermal annealing decreases some of the defects and internal surfaces, which decreases the extent of phonon scattering and $\kappa$ increases. There is usually a very small decrease in the specific heat also, with little effect on $\kappa$. Decrease of any structural fault and defect on isothermal and/or heat annealing would increase $\kappa$ of ice $\mathrm{I}_{\mathrm{c}(\mathrm{h})}^{\mathrm{sd}}$ and ice Ih samples.

Our findings of the magnitude of $\kappa$ suggest that, in addition to stacking faults, other types of disorder are present and that the sum of all types of disorder gradually decreases on heating ice $\mathrm{I}_{\mathrm{c}(\mathrm{h})}^{\mathrm{sd}}$. As $\kappa$ remains almost constant, or increases slightly, when ice $\mathrm{I}_{\mathrm{c}(\mathrm{h})}^{\mathrm{sd}}$ made from HDA is heated in the range $150 \mathrm{~K}-220 \mathrm{~K}$, it means that the increase in three phonon umklapp scattering ${ }^{73}$ is roughly compensated by a decrease in the structural disorder. Therefore, part of the difference between $\kappa$ of ice $I_{\mathrm{ch}}^{\mathrm{sd}}$ and Ih may be due to their different crystal structures, and part due to stacking-disorder and other type of disorder in the samples of ice $\mathrm{I}_{\mathrm{c}(\mathrm{h})}^{\mathrm{sd}}$. The difference between their structures may raise $\kappa$ or lower it, but the difference in terms of an increased extent of a disorder always lowers $K$ and changes $d \kappa / d T$.

Kuhs et al. ${ }^{31}$ established a quantitative link between the crystallite size established by diffraction and electron microscopic images of the material. With progressive annealing, the crystallite size evolved from several nanometers into the micrometer range, with isometric nature of the crystallites, but with markedly rough surfaces parallel to the stacking direction. Their studies could not include the effect of pressure, which, if it is anisotropic, would lead to deformation flaws in the sample, and hence to the possibility of formation or annihilation of stacking faults. ${ }^{40,44,45}$ So, we now compare the $\kappa$ of the ice $\mathrm{I}_{\mathrm{c}(\mathrm{h})}^{\mathrm{sd}}$ samples made by heating ice II under pressure. In our experiments performed on heating, $\kappa$ changes with time and $T$. We interpret it as loss of stacking and microstructural disorders in ice $\mathrm{I}_{\mathrm{c}(\mathrm{h})}^{\mathrm{sd}}$. Since $\kappa$ of ice $\mathrm{I}_{\mathrm{c}(\mathrm{h})}^{\mathrm{sd}}$ made from ice II is $8 \%-11 \%$ higher than $\kappa$ of ice $\mathrm{I}_{\mathrm{c}(\mathrm{h})}^{\mathrm{sd}}$ made from HDA at $200 \mathrm{~K}$, the latter ice $\mathrm{I}_{\mathrm{c}(\mathrm{h})}^{\mathrm{sd}}$ is more disordered than the former, but the difference becomes less on heating. We also note that $\kappa$ of ice $\mathrm{I}_{\mathrm{c}(\mathrm{h})}^{\text {sd }}$ made by heating ice II to $200 \mathrm{~K}$ was thermally reversible at $T<200 \mathrm{~K} .{ }^{19}$ So, this ice $\mathrm{I}_{\mathrm{c}(\mathrm{h})}^{\text {sd }}$ is stable against further loss of microstructural details and stacking disorder, Hansen et al. ${ }^{57,71}$ wrote that different precursors produce ice $I_{c(h)}^{\text {sd }}$ with different diffraction details and annealing effects and transformation to ice Ih occurs cleanly (without retention of disorder). They ${ }^{71}$ reported "particle sizes" in the range 250-700 $\AA$ for ice $\mathrm{I}_{\mathrm{c}(\mathrm{h})}^{\mathrm{sd}}$ 
made by recrystallizing ice $\mathrm{V}$, and similar sizes for ice $\mathrm{I}_{\mathrm{c}(\mathrm{h})}^{\mathrm{sd}}$ made from IX, all at ambient pressure. Our samples formed by recrystallization at high $p$ and studied at high $p$ are compact and unlikely to contain $\mathrm{nm}$-size crystallites. We also note that scanning electron micrographs of the frost of ice $\mathrm{I}_{\mathrm{c}(\mathrm{h})}^{\mathrm{sd}}{ }^{31}$ have shown that its crystallites are in the 4-200 $\mathrm{nm}$ size range, and they grow by at least a factor of two on annealing before transforming to ice $\mathrm{Ih}$. These $\mathrm{nm}$-size crystallites which are partly separated by voids ${ }^{31}$ would have a large surface energy relative to the volume energy. This would not only increase their vapor pressure but also affect formation of stacking faults, because such faults terminate at the surface of a crystallite. The size of the crystallites of ice $\mathrm{I}_{\mathrm{c}(\mathrm{h})}^{\mathrm{sd}}$ formed on freezing emulsified droplets of $0.9 \mu \mathrm{m}$ size at $\sim 231.7 \mathrm{~K}$ is not known, nor is it known how much do they grow on heating before transforming to ice $\mathrm{Ih}^{36}$

We return to discuss the $\kappa-T$ plots in Fig. 1. These show three features: (i) at different $p$, ice $\mathrm{I}_{\mathrm{c}(\mathrm{h})}^{\mathrm{sd}}$ of different $\kappa$ is produced, and this ice $\mathrm{I}_{\mathrm{c}(\mathrm{h})}^{\mathrm{sd}}$ sharply transforms to ice $\mathrm{Ih}$, (ii) on extrapolation, $k$ of the differently formed ice $\mathrm{I}_{\mathrm{c}(\mathrm{h})}^{\mathrm{sd}} \mathrm{s}$ approaches the same value near $220 \mathrm{~K}$, which is the temperature of the ice $\mathrm{I}_{\mathrm{c}(\mathrm{h})}^{\mathrm{sd}}$ to ice Ih transition observed here, and, (iii) higher $p$ and lower $q(70 \mathrm{MPa}, 6 \mathrm{~K} / \mathrm{h})$ of HDA produce ice $\mathrm{I}_{\mathrm{c}(\mathrm{h})}^{\mathrm{sd}}$ of higher $\kappa$ than $(40 \mathrm{MPa}) 7 \mathrm{~K} / \mathrm{h}$ and $(50 \mathrm{MPa}) 12 \mathrm{~K} / \mathrm{h}$ which indicates that the first of the three samples is less disordered than the other two. If the decrease in $\kappa$ were entirely due to the presence of stacking faults, there would be less such faults in ice $\mathrm{I}_{\mathrm{c}(\mathrm{h})}^{\mathrm{sd}}$ formed by heating HDA at $70 \mathrm{MPa}$ at $6 \mathrm{~K} / \mathrm{h}$, than in the other two ice $\mathrm{I}_{\mathrm{c}(\mathrm{h})}^{\mathrm{sd}}$ samples, but heating of vapor deposited, and droplets-frozen ice $\mathrm{I}_{\mathrm{c}(\mathrm{h})}^{\mathrm{sd}}$ converts ice Ic sequences to ice Ih sequences or decreases, $n_{\mathrm{Ic} \text {, seq }}$ or $\chi \cdot^{31,71}$ This indicates that at $T$ near $140 \mathrm{~K}$, a lower $q$ converts cubic sequences to hexagonal sequences in ice $\mathrm{I}_{\mathrm{c}(\mathrm{h})}^{\mathrm{sd}}$, or decreases $n_{\mathrm{Ic}, \text { seq }}$ and $\chi$ more than a higher $q$. The effect of pressure on $\chi$ seems less certain, because, as in all other studies, our measurements were performed in a non-equilibrium state, where the time-temperature protocols change $\kappa \cdot{ }^{74}$ For the sample at $50 \mathrm{MPa}$, HDA to LDA transformation occurs too fast to be obvious in Fig. 1, but the ice $\mathrm{I}_{\mathrm{c}(\mathrm{h})}^{\mathrm{sd}}$ produced has a higher $\kappa$.

Two of our ice $I_{c(h)}^{\text {sd }}$ samples that were formed by heating HDA have $\kappa$ values significantly different from the third sample. This difference may not be explained by different $\chi$ resulting from different $q$, since Malkin et al. ${ }^{36}$ found only $2 \%$ difference in "cubicity" when $q$ differed by a factor of five $\left(0.1\right.$ and $\left.0.5 \mathrm{~K} \mathrm{~min}^{-1}\right)$, and $q$ in our study differs by factor of two $\left(0.1-0.2 \mathrm{~K} \mathrm{~min}^{-1}\right)$. Also, their observed difference ${ }^{36}$ in "cubicity" can be partly a consequence of a higher $q$ pushing the transition to higher $T$. In addition to the transition temperatures for HDA and LDA being different at different $p$, there are other effects: (i) ice Ic in LDA seems to nucleate at a higher $T$ than ice Ic in HDA, (ii) the mechanism of nucleation of ice Ic in these solids differs from that of nucleation in supercooled nm-size droplets in emulsions, and (iii) as shown by the authors of Refs. 31 and 36, who used diffraction methods, the micro-structures of the precursor phases, namely, ices II, V, IX, and LDA and HDA, and nm-size water droplets affect the $\chi$ values of the ice $\mathrm{I}_{\mathrm{c}(\mathrm{h})}^{\mathrm{sd}}$.

\section{B. Thermal conductivity, stacking disorder, pressure, and annealing effects}

We now consider the effect of pressure on $\chi$. For the usual condition in which $n_{\mathrm{Ic}, \text { seq }}>n_{\mathrm{Ih} \text {, seq }}$, or $\chi>0.5$, the net change in $\kappa$ with $p$ may be written as

$$
\left(\frac{\partial \kappa}{\partial p}\right)_{T}=\left(\frac{\partial \kappa}{\partial p}\right)_{\chi}+\left(\frac{\partial \kappa}{\partial \chi}\right)_{T}\left(\frac{\partial \chi}{\partial p}\right)_{T}
$$

If $\kappa$ were to decrease with decrease in $\chi$ from its $100 \%$ value (ice Ic becoming ice $\left.\mathrm{I}_{\mathrm{c}(\mathrm{h})}^{\mathrm{sd}}\right)$, the term $(\partial \kappa / \partial \chi)_{T}$ would be positive. There is no evidence that $\chi$ changes or is expected to change with $p$. Therefore, $(\partial \chi / \partial p)_{T}$ may be 0 , and the second term on RHS of Eq. (1) may be zero. Thus, the term $(\partial \kappa / \partial p)_{T}=(\partial \kappa / \partial p)_{\chi}$. The measured $(\partial \kappa / \partial p)_{T}$ for ice $\mathrm{I}_{\mathbf{c}(\mathrm{h})}^{\mathrm{sd}}$ at $129 \mathrm{~K}$ from Fig. 2 is $-1.2 \mathrm{~W} \mathrm{~m}^{-1} \mathrm{~K}^{-1}$ per GPa in the $p$ range up to $150 \mathrm{MPa}$. Since our sample was formed by heating ice II at $50 \mathrm{MPa}$ at a slow rate of about $0.3 \mathrm{~K} \mathrm{~min}^{-1}$ which is known to produce ice $\mathrm{I}_{\mathrm{c}(\mathrm{h})}^{\mathrm{sd}}$ with cubicity of $\sim 70 \%$ at ambient conditions (Table IV, Ref. 36), $n_{\mathrm{Ic}, \text { seq }}$ or $\chi$ of our sample would be close to $70 \%$. How the $(\partial \kappa / \partial p)_{T}$ value would change with change in $\chi$ is not known. But it is known that if we introduce disorder in the structure of a crystal by any means, or amorphize it, $\kappa$ decreases and $(\partial \kappa / \partial p)_{T}$ become positive, i.e., $(\partial \kappa / \partial p)_{T}$ of fully disordered solid is positive. (LDA being a known exception. ${ }^{70}$ )

In the phase diagram of high pressure phases of ice, one finds that whatever the $T, p$ condition used, ice $\mathrm{I}_{\mathrm{c}(\mathrm{h})}^{\mathrm{sd}}$ is always produced in the stability region of another phase, and at ambient pressure it forms obviously in the domain of ice Ih. Furthermore, ice Ic and ice $\mathrm{I}_{\mathrm{c}(\mathrm{h})}^{\mathrm{sd}}$ neither melt (to produce water) nor transform reversibly to another solid. Obviously, ice Ic with or without stacking disorder is not one of the stable phases of ice under any $T, P$ condition, and that makes it difficult to regard the stacking disordered ice I as a distinct phase.

When the applied pressure is non-hydrostatic, crystals plastically deform. The deformation increases the self-diffusion rate, but whether or not plastic deformation of the ices increases the Bjerrum defect diffusion is unknown. Also, the ice Ih surface, like the crystal surfaces in general, is known to be disordered (liquid-like) and to have higher energy than the bulk. Moreover, when water crystallizes to form fine ice Ih crystals, a detectably significant amount of liquid water persists at the grain boundaries and grain junctions of the ice Ih crystals, ${ }^{74,75}$ and this amount decreases at least in thermodynamic terms as $T$ is decreased. Also, nanometer-size water droplets can coexist with cubic ice particles of about the same size at temperatures in the $150-180 \mathrm{~K}$ range. ${ }^{76}$

Since $\kappa$ of water is lower than that of ice, the measured $\kappa$ of a polycrystalline sample of ice $\mathrm{I}_{\mathrm{c}(\mathrm{h})}^{\mathrm{sd}}$ would be lower when the crystal size is in the $\mu \mathrm{m}$ to $\mathrm{nm}$ range (with liquid water at grain junctions) than when crystal size is larger. Also, a decrease in the crystal sizes increases the phonon scattering from grain boundaries, and the transformation temperature of the cubic ice crystals to ice $\mathrm{Ih}, T_{t}$, would vary with their size according to the Gibbs-Thomson's equation. ${ }^{77-79}$ Thus, $k$ of a microcrystalline sample and the $T$-range over which its $\kappa$ can be measured would depend not only upon $\chi$ but also the crystal size in the $\mu \mathrm{m}$ to $\mathrm{nm}$ range. From the transformation 
exotherms, $T_{t}$ was found to be in the 190-220 K range. ${ }^{11,62,80}$ Since $T_{t}$ here does not vary with the heating rate, we suggest that the 190-200 K range indicates crystal-size effect, with a corresponding effect on $\kappa$.

Experiments have shown that thermal annealing converts ice Ic sequences gradually to ice Ih sequences in ice $\mathrm{I}_{\mathrm{c}(\mathrm{h} h}^{\text {sd }}$, but it does not convert ice Ih sequences to ice Ic sequences. If ice $\mathrm{I}_{\mathrm{c}(\mathrm{h})}^{\mathrm{sd}}$ of unknown $\chi$ value was heated, $\chi$ or $n_{\mathrm{Ic} \text {, seq }}$ would decrease at a rate that depends upon $q, T$, and $p$. One expects that if ice $\mathrm{I}_{\mathrm{c}(\mathrm{h})}^{\mathrm{sd}}$ is a heated at a rate $q$ up to a temperature $T_{1}$ and its energy decreases, $\chi$ would decrease to a value say $\chi_{1}$ at $T_{1}$ (more ice Ic sequences convert to ice Ih sequences) and if it is then immediately cooled to say $77 \mathrm{~K}, \chi_{1}$ would become kinetically frozen. If an ice $\mathrm{I}_{\mathrm{c}(\mathrm{h})}^{\mathrm{sd}}$ sample is heated at the same $q$ to a higher $T$, say $T_{2}, \chi$ would decrease to $\chi_{2}$ (with even more ice Ih sequences), and $\chi_{2}$ would become kinetically frozen at say $77 \mathrm{~K}$. In this case, $\chi_{2}<\chi_{1}$. Thus, samples of different $\chi$ may be made for studies by diffraction and other methods.

\section{General aspects of stacking disorder in interlaced ice Ic and ice Ih sequences}

Kohl et al. ${ }^{81}$ had found that annealing of $150 \mathrm{~K}$ deposits for $5 \mathrm{~min}$ at $183 \mathrm{~K}$ and also for $5 \mathrm{~min}$ at $193 \mathrm{~K}$, increased the (Xray diffractogram) stacking-fault peak height and decreased its width, thus making the stacking fault peak appear sharper, and there were corresponding changes in the exothermic feature at $190 \mathrm{~K}$. They also found that the stacking-fault peak of almost the same intensity appeared for ice $\mathrm{I}_{\mathrm{c}(\mathrm{h})}^{\mathrm{sd}}$ (they used the term ice Ic with stacking faults) made by annealing hyperquenched glassy water $(\mathrm{HGW})$ at $183 \mathrm{~K}$, and of ice $\mathrm{I}_{\mathrm{c}(\mathrm{h})}^{\mathrm{sd}}$ made by heating the sintered state of vapor-deposited amorphous solid water (ASW). Thus, their studies indicated that, (i) the stacking fault peak is not always much less than the (111) peak, (ii) annealing increases the stacking fault peak height significantly, and, (iii) there is little difference between the stacking fault peaks of ice $\mathrm{I}_{\mathrm{c}(\mathrm{h})}^{\mathrm{sd}}$ samples made by heating HGW, and ASW.

Kuhs et al. ${ }^{31}$ found that when ice $\mathrm{I}_{\mathrm{ch}}^{\mathrm{sd}}$ produced by heating the vapor-deposited frost was annealed isothermally at $175 \mathrm{~K}$, $180 \mathrm{~K}, 185 \mathrm{~K}$, or $190 \mathrm{~K}$ (Fig. 3 in Ref. 31), cubicity, $\chi$, or $n_{\mathrm{Ic}, \text { seq }}$ decreased with the annealing time more rapidly at a higher $T$ than at a lower $T$. To compare it against the transformation of ice II to ice $\mathrm{I}_{\mathrm{c}(\mathrm{h})}^{\mathrm{sd}}$ in the study of Malkin et al., ${ }^{36}$ when ice II was heated slowly, i.e., more time was allowed for its transformation to ice $\mathrm{I}_{\mathrm{c}(\mathrm{h})}^{\mathrm{sd}}$, the ice $\mathrm{I}_{\mathrm{c}(\mathrm{h})}^{\mathrm{sd}}$ sample formed at $165 \mathrm{~K}$ had a higher $\chi$, i.e., $n_{\mathrm{Ic}, \mathrm{seq}}$ increased. But when more time was allowed, ice $\mathrm{I}_{\mathrm{c}(\mathrm{h})}^{\mathrm{sd}}$ formed from the vapor phase to anneal isothermally at $T$ of $175 \mathrm{~K}, 180 \mathrm{~K}, 185 \mathrm{~K}$, or $190 \mathrm{~K}, \chi$ or $n_{\text {Ic, seq }}$ decreased further. ${ }^{31}$ This indicates that the mechanism of transformation from ice II to ice $\mathrm{I}_{\mathrm{c}(\mathrm{h})}^{\mathrm{sd}}$ does not include the mechanism that decreases $\chi$ of ice $\mathrm{I}_{\mathrm{c}(\mathrm{h})}^{\mathrm{sd}}$ formed on heating ice II, or that the ice II's transformation mechanism competes with the mechanism that decreases $\chi$. Alternatively, vapordeposited ice $\mathrm{I}_{\mathrm{c}(\mathrm{h})}^{\mathrm{sd}}$ differs from the ice $\mathrm{I}_{\mathrm{c}(\mathrm{h})}^{\mathrm{sd}}$ made by heating ice II. It should be noted that ices II, ${ }^{16}$ ice VII,${ }^{33}$ and XII ${ }^{34}$ become amorphous solids on isothermal annealing or rate heating before transforming to ice $\mathrm{I}_{\mathrm{c}(\mathrm{h})}^{\mathrm{sd}}$. Heating of such low-density amorphs converts them to ultraviscous water, which then crystallizes to ice $\mathrm{I}_{\mathrm{c}(\mathrm{h})}^{\mathrm{sd}}{ }^{26}$ So, except for the vapor-deposited and decomposed $\mathrm{CH}_{4}$ and $\mathrm{CO}_{2}$ clathrate hydrates in which the particle size is on the order of tens of nm, most samples of ice $I_{c(h)}^{s d}$ seem to have been formed by starting with bulk water freezing to ice Ih and then pressurizing it to produce high pressure crystalline ices or by collapsing the structures of ice Ih, Ic, or ice $\mathrm{I}_{\mathrm{c}(\mathrm{h})}^{\mathrm{sd}}$ under $1 \mathrm{GPa}$ pressure and then heating all at ambient pressure. More quantitative studies ${ }^{31,35}$ have shown that different high pressure ices yield different stacking fault features in diffraction studies and hence, ice $\mathrm{I}_{\mathrm{c}(\mathrm{h})}^{\mathrm{sd}}$ obtained by heating high pressure crystalline ices shows a topotactic relationship between the parent phase and ice $\mathrm{I}_{\mathrm{c}(\mathrm{h})}^{\mathrm{sd}}$. (Topotactic means a structural change to a crystalline solid such that the final lattice is related to that of the original material by one or more crystallographically equivalent, orientational relationships.) This interesting relationship may need further support from studies by other techniques.

Neutron or X-ray diffraction of ice $\mathrm{I}_{\mathrm{c}(\mathrm{h})}^{\mathrm{sd}}$ made by vapor deposition, by ex situ recrystallizing ices II, IV, V, III, or IX at ambient pressure, by crystallizing LDA and HDA and by decomposing $\mathrm{CO}_{2}$ and $\mathrm{CH}_{4}$ clathrates have shown different amounts of stacking faults, ${ }^{36}$ and there is no unique solid which could be given the name ice $I_{s d}$ in the terminology of Malkin et al. ${ }^{36}$ Like a topologically disordered solid, there are numerous ice $\mathrm{I}_{\mathrm{c}(\mathrm{h})}^{\mathrm{sd}} \mathrm{s}$, each made by a different method and precursor, namely, an amorph, supercooled water, gel, aqueous solution, and vapor deposition. A slow decrease in "cubicity" from loss of cubic sequences was observed on annealing of vapor-deposited frost at $175 \mathrm{~K}$; it became rapid at $185 \mathrm{~K}$ and a smaller, further change occurred on annealing it at $190 \mathrm{~K}$ (Fig. 3, Ref. 31), as mentioned above. This shows the temporal instability of ice $\mathrm{I}_{\mathrm{c}(\mathrm{h})}^{\mathrm{sd}}$. Since the process of decrease in $\chi$ and the ice $\mathrm{I}_{\mathrm{c}(\mathrm{h})}^{\mathrm{sd}}$-ice Ih transformation temperature itself is irreversible, one cannot define a phase boundary for ice $\mathrm{I}_{\mathrm{c}(\mathrm{h})}^{\mathrm{sd}}$. In the phase diagram of thermodynamically stable ices, neither ice $I_{c(h)}^{\text {sd }}$ appears at ambient pressure, nor do the solids from which it was made.

Also only droplets of $<0.9 \mu \mathrm{m}$ size in an emulsion (water, lanolin, and mineral oil) froze to ice $\mathrm{I}_{\mathrm{c}(\mathrm{h})}^{\mathrm{sd}}$ at $231.7 \mathrm{~K},{ }^{8}$ a temperature $12 \mathrm{~K}$ higher than the $T(220 \mathrm{~K})$ at which ice $\mathrm{I}_{\mathrm{c}(\mathrm{h})}^{\mathrm{sd}}$ transforms to ice $\mathrm{Ih}$ at the heating rate $q$ of $0.1 \mathrm{~K} \mathrm{~min}^{-1}$ (Fig. 1), $\sim 14 \mathrm{~K}$ higher than the $T$ at which it transforms to ice Ih at $q$ of $10 \mathrm{~K} / \mathrm{h},{ }^{60}$ and $\sim 72 \mathrm{~K}$ higher than $T$ at which it begins to transform to ice $\mathrm{Ih}$ at $q$ of $30 \mathrm{~K} \mathrm{~min}^{-1} .{ }^{1}$ Emulsified droplets of $<0.9 \mu \mathrm{m}$ size freeze to form $40-200 \mathrm{~nm}$ size ice $\mathrm{I}_{\mathrm{c}(\mathrm{h})}^{\mathrm{sd}}$ crystallites, and droplets of $>0.9 \mu \mathrm{m}$ size freeze to form ice Ih. The apparent upper limit of $0.9 \mu \mathrm{m}$ droplets size for forming ice $\mathrm{I}_{\mathrm{c}(\mathrm{h})}^{\mathrm{sd}}$ indicates that the ratio of the interfacial area between the lanolin and water (and the $\mathrm{H}$-bond interaction between the two) to the volume of the droplets is of relevance in forming ice $\mathrm{I}_{\mathrm{c}(\mathrm{h})}^{\mathrm{sd}}$ in emulsified droplets.

In summary, we studied bulk samples of ice $\mathrm{I}_{\mathrm{c}(\mathrm{h})}^{\mathrm{sd}}$ and ice Ih, which differ from those of other studies, ${ }^{8,31,36,37}$ and our samples were heated or cooled in situ at $30 \mathrm{~K} / \mathrm{h}$, or at a lower rate, at $p>0.1 \mathrm{MPa}$. LDA transforms to ultraviscous water around $136 \mathrm{~K}$ and the ultraviscous water ${ }^{26}$ transforms to ice $\mathrm{I}_{\mathrm{c}(\mathrm{h})}^{\mathrm{sd}}$ beginning at $\sim 148 \mathrm{~K}$. On heating at $0.1 \mathrm{MPa}$, the recovered state of some high pressure crystalline ices first became amorphous and then transformed to ice $\mathrm{I}_{\mathrm{c}(\mathrm{h})}^{\mathrm{sd}}$. Ex situ 
heating of ice XII at ambient pressure has also produced ice $\mathrm{I}_{\mathrm{c}(\mathrm{h})}^{\mathrm{sd}}$, and recent studies have shown that on annealing isothermally at $120 \mathrm{~K}$, ice XII transforms to amorphous ice. ${ }^{34}$ This would also indicate that ice $\mathrm{I}_{\mathrm{c}(\mathrm{h})}^{\mathrm{sd}}$ forms on freezing of ultraviscous water, not always by solid-solid transformation on heating of high pressure ices. Vapor-deposition on a cold substrate produces ice $\mathrm{I}_{\mathrm{c}(\mathrm{h})}^{\mathrm{sd}}$ and ice $\mathrm{I}_{\mathrm{c}(\mathrm{h})}^{\mathrm{sd}}$ is also formed by decomposing clathrate hydrates.

\section{CONCLUSIONS}

Thermal conductivity, $\kappa$, of ice $\mathrm{I}_{\mathrm{c}(\mathrm{h})}^{\mathrm{sd}}$ formed by recrystallization of ice II is $\sim 20 \%$ less than that of ice Ih. When HDA converts to ice $\mathrm{I}_{\mathrm{c}(\mathrm{h})}^{\mathrm{sd}}$ on heating, $\kappa$ tends to increase with increase in $T$ until ice $\mathrm{I}_{\mathrm{c}(\mathrm{h})}^{\mathrm{sd}}$ transforms sharply to ice Ih at $220 \mathrm{~K}$. When ice II converts to ice $\mathrm{I}_{\mathrm{c}(\mathrm{h})}^{\mathrm{sd}}$ on heating, $\kappa$ increases through the $170-210 \mathrm{~K}$ range, and thereafter cooling and heating in the $120-170 \mathrm{~K}$ range does not change $\kappa$, as seen in Fig. 1. When $\chi<0.5$, i.e., the condition is that $n_{\mathrm{Ic}, \mathrm{seq}}<n_{\mathrm{Ih} \text {, seq }}$ or the stacking faults are cubic sequences in ice Ih, loss of cubic sequences increases $\kappa$. Decrease in the surface area of micro-crystals, in the population of point defects, of dislocations and grain-boundary defects also increases $\kappa$. The pressure dependence of $\kappa$ of ice $\mathrm{I}_{\mathrm{c}(\mathrm{h})}^{\mathrm{sd}}$ at $p$ up to $150 \mathrm{MPa}$ is $-1.2 \mathrm{~W} \mathrm{~m}^{-1} \mathrm{~K}^{-1}$ per $\mathrm{GPa}$, which is about the same as that of ice Ih within experimental errors.

In addition to lowering $\kappa$, we suggest that stacking faults in ice Ic would have at least four experimentally testable consequences, (i) ice $\mathrm{I}_{\mathrm{c}(\mathrm{h})}^{\mathrm{sd}}$ containing more of such faults would show a greater distribution of dielectric and mechanical relaxation times than one containing less faults, (ii) ice $\mathrm{I}_{\mathrm{c}(\mathrm{h})}^{\mathrm{sd}}$ would be more difficult to deform than ice Ih, because of the hindrance to dislocation movement across the stacking faults, and (iii) protons in ice $\mathrm{I}_{\mathrm{c}(\mathrm{h})}^{\mathrm{sd}}$ would not order in the same way as in ice Ih. This latter would be the case for samples in which $n_{\text {Ic, seq }}$ value is high. (iv) the pressure needed to collapse ice $I_{c(h)}^{\text {sd }}$ would vary with the extent of stacking disorder or $\chi$. The previously found lower pressure ${ }^{70}$ for collapse of ice Ic or ice $\mathrm{I}_{\mathrm{c}(\mathrm{h})}^{\mathrm{sd}}$ to HDA relative to that of ice Ih probably indicates it. It would be interesting to investigate if the ice $I_{c(h)}^{\text {sd }}$ that forms on heating HDA is identical to the ice $\mathrm{I}_{\mathrm{c}(\mathrm{h})}^{\mathrm{sd}}$ whose collapse had produced HDA.

Scanning electron micrographs ${ }^{72,82}$ have shown that morphology of the ice $\mathrm{I}_{\mathrm{c}(\mathrm{h})}^{\mathrm{sd}}$ crystallites is trigonal (space group $P 3 m 1$ ) or pseudo-hexagonal, and pseudo-hexagonal shape cannot be taken as evidence of ice Ih. (Hallet et al. ${ }^{7}$ had previously shown images of atmospheric ice crystals with three fold rotational symmetry. Trigonal shape ice crystals have also been found to occur over a wide range of atmospheric condition, and in troposphere and stratosphere. ${ }^{82}$ ) To establish that ice $\mathrm{I}_{\mathrm{c}(\mathrm{h})}^{\mathrm{sd}}$ is a new phase, one should obtain its proton (or orientationally) ordered phase derived from $P 3 m 1$ space group. Variation in $\chi$ among different samples of ice $I_{c(h)}^{\text {sd }}$ is reminiscent of crystals containing different population of dislocations that anneal out on heating with loss in enthalpy and are not reproduced by cooling the heat-annealed sample. Different preparation methods ${ }^{31,35}$ yield different ice $\mathrm{I}_{\mathrm{c}(\mathrm{h})}^{\mathrm{sd}}$ characterized by different $\chi$ and experimentally distinguished by the relative intensity of their diffraction peaks, thereby suggesting a topotactic relationship between the parent phase and ice $\mathrm{I}_{\mathrm{c}(\mathrm{h})}^{\mathrm{sd}}$ in Ref. 31 . This may also indicate indirectly that there is a continuity of stacking disorder that depends upon the parent phase. It is conceivable that vapor deposition of water at different rates may produce ice $\mathrm{I}_{\mathrm{c}(\mathrm{h})}^{\mathrm{sd}}$ with different extent of stacking faults, again indicating a continuity of disordered structure from interlacing of cubic and hexagonal sequences in the suggested transformation, ice Ic $\rightarrow$ ice $\mathrm{I}_{\mathrm{c}(\mathrm{h})}^{\mathrm{sd}} \rightarrow$ ice $\mathrm{I}_{\mathrm{ch}}^{\mathrm{sd}} \rightarrow$ ice $\mathrm{I}_{(\mathrm{c}) \mathrm{h}}^{\mathrm{sd}}$ $\rightarrow$ ice Ih, which includes one more step, Ic $\rightarrow$ ice $I_{c(h)}^{s d}$, than those given in Ref. 71.

Cubic and hexagonal ices are regarded as polytypes. According to Guinier et al., ${ }^{83}$ polytypes are structural modifications, and each modification may be regarded as built up by stacking sequences of (nearly) identical structure and composition differing only in their stacking sequence. Because of the multiplicity of crystals with varying fraction of hexagonal sequences, ice $\mathrm{I}_{\mathrm{c}(\mathrm{h})}^{\mathrm{sd}}$ polytype itself may have varying properties. Because of these intrinsic differences, we argue that the effect of stacking disorder may be better ascertained by studying the properties of ice $\mathrm{I}_{\mathrm{c}(\mathrm{h})}^{\mathrm{sd}}$ containing different fractions of ice Ih sequences (sequences) than by studying the difference between the properties of ice $\mathrm{I}_{\mathrm{c}(\mathrm{h})}^{\mathrm{sd}}$ and $\mathrm{Ih}$, and ignoring the possibility that ideal ice Ic may exist.

Although the existence of stacking disorder in metals and its effect on a metal's properties have been known for a long time, and neutron diffraction data published by Kuhs et al. ${ }^{37}$ demonstrated stacking disorder in vapor-deposited cubic ice in 1987 , interest in the subject has grown only recently. ${ }^{31,36,71}$ However, the information obtained has been almost entirely based upon the X-ray and neutron diffraction data. It is not known how the presence of stacking faults affects the lattice spacing, local strain, and hence, the energy from incoherent interfaces of small particles. ${ }^{80}$ One expects that the arrangement of $\mathrm{H}_{2} \mathrm{O}$ molecules at the grain boundaries of the ice $\mathrm{I}_{\mathrm{c}(\mathrm{h})}^{\mathrm{sd}}$ crystals would be topologically disordered. Since the crystal size is already small, there would be a relatively large, time-dependent, grain boundary area whose structure would determine some of the properties of ice $\mathrm{I}_{\mathrm{c}(\mathrm{h})}^{\mathrm{sd}}$, which may be investigated. Since disordered solids are known to be more common than the ordered solids (all crystals at $T>0 \mathrm{~K}$ have disorder due to the presence of point defects in their structure), it would be of interest to determine if stacking disorder could be regarded as another type of disorder different from those from the point defects.

For further studies of the effects of stacking disorder on the properties of ice I, it would be important to develop a technique for reproducibly introducing stacking faults in an ice lattice by deformation or by other methods of adding energy, and to determine if this disorder plays a role in, (i) the ordering transformation of ice Ih to ice XI, and (ii) the collapse of ice Ih and Ic at high pressures which seems to be crystal-grain size dependent. ${ }^{84}$ Two dimensional layered structure of hydrogen bonded water molecules is often found in some crystal hydrates such as $\mathrm{SnCl}_{2} \cdot 2 \mathrm{H}_{2} \mathrm{O}$ (see discussion in Ref. 85), occasionally referred to as two-dimensional protonic conductors. It may occur in mono-layers of $\mathrm{H}_{2} \mathrm{O}$ adsorbed on a substrate. Intercalation compounds formed by inserting ions between graphite layers may also show stacking faults of the 
graphite layers. It is conceivable that stacking disorder occurs in nanometer and $\mu \mathrm{m}$ size ice crystals formed on rapid cooling of biological and synthetic materials to low temperatures, and that this disorder may limit their crystal-growth. It remains to be investigated if the requirement that two crystal lattices have the same density and nearly the same energy to form stacking faults can be fulfilled by some structures formed by carbon atoms alone. We also note a recent finding that twinning and/or stacking faults may be present ${ }^{86}$ in meteoritic-impact shock wave-produced (and also laboratory-produced) hexagonal diamond known as Lonsdaleite. They ${ }^{86}$ observed the $\{113\}$ twins and/or $\{111\}$ stacking faults over a $2 \mathrm{~nm}$ scale by using ultra-high resolution electron microscope images. ${ }^{86}$ Their findings seems intriguing because the density of Lonsdaleite is $3.2-3.52 \mathrm{~g} / \mathrm{cm}^{3}$, [http://www.handbookofmineralogy.com/ pdfs/lonsdaleite.pdf], which is comparable to the measurable density of $3.514 \mathrm{~g} / \mathrm{cm}^{3}$ of (cubic) diamond. (Theoretical density of Lonsdaleite is $3.51 \mathrm{~g} / \mathrm{cm}^{3}$, which is the same as the measured density of $3.514 \mathrm{~g} / \mathrm{cm}^{3}$ of cubic diamond. Densities of graphite and graphene, $2.09-2.23 \mathrm{~g} / \mathrm{cm}^{3}$, are much less than that of Lonsdaleite.) The densities of cubic and hexagonal ice are identical within experimental errors. Also, zirconium metal exists in both the cubic and hexagonal forms, ${ }^{87}$ whether it too forms stacking faults is yet to be studied in detail. Since stacking faults constitute a type of disorder at nanometer scale in crystals, there is need to study how the methods used for forming these crystals (from the vapor, liquid, or solid), the pressure and temperature conditions during their formation, the presence of impurities, interfaces, pressure gradients, and shear deformation affect the extent of this disorder. This is important not only for the occurrence of stacking disorder in naturally occurring cubic ice and formation of cubic ice in cryo-preserved biomaterials, but also for technologically important, natural, and manufactured materials whose properties are significantly affected by such a disorder.

\section{ACKNOWLEDGMENTS}

This work was supported by the Swedish Research Council, Grant No. 621-2012-3336. We acknowledge financial support for equipment from Carl Trygger Foundation and Magnus Bergvalls foundation.

${ }^{1}$ A. Hallbrucker and E. Mayer, Nature 325, 601 (1987).

${ }^{2}$ G. P. Johari, A. Hallbrucker, and E. Mayer, J. Phys. Chem. 93, 4986 (1989).

${ }^{3}$ D. C. Steytler, J. C. Dore, and C. J. Wright, J. Phys. Chem. 87, 2458 (1983).

${ }^{4}$ A. Fouzri, R. Dorbez-Sridi, and M. Oumezzine, J. Chem. Phys. 116, 791 (2002).

${ }^{5}$ K. Morishige, H. Yasunaga, and H. Uematsu, J. Phys. Chem. C 113, 3056 (2009).

${ }^{6}$ D. M. Murphy, Geophys. Res. Lett. 30, 2230 doi:10.1029/2003GL018566 (2003).

${ }^{7}$ J. Hallet, W. P. Arnott, M. P. Bailey, and J. T. Hallet, in Cirrus, edited by D. K. Lynch, K. Sassen, D. O. Starr, and G. Stephens (Oxford University Press, New York, 2002), pp. 41-77.

${ }^{8}$ B. J. Murray and A. K. Bertram, Phys. Chem. Chem. Phys. 8, 186 (2006).

${ }^{9}$ E. Whalley, Science 211, 389 (1981).

${ }^{10}$ E. Whalley, J. Phys. Chem. 87, 4174 (1983).

${ }^{11}$ H. P. Barendrecht, Z. Phys. Chem. 20, 234 (1896).

${ }^{12}$ J. Dewar, Chem. News 91, 216 (1905).

${ }^{13}$ E. F. Burton and W. F. Oliver, Proc. R. Soc. London A 153, 166 (1935).

${ }^{14}$ R. L. McFarlan, Phys. Rev. 49, 637 (1936).

${ }^{15}$ H. König, Z. Kristallogr.-Cryst. Mater. 105, 279 (1943).
${ }^{16}$ J. E. Bertie, L. D. Calvert, and E. Whalley, J. Chem. Phys. 38, 840 (1963). They noted that the diffractogram of ice II during its annealing showed blurred spots before ice II transformed to ice Ic. The observation indicates that an ice-amorph first formed on annealing at 1 bar pressure.

${ }^{17}$ J. E. Bertie, L. D. Calvert, and E. Whalley, Can. J. Chem. 42, 1373 (1964).

${ }^{18}$ G. P. Arnold, E. D. Finch, S. W. Rabideau, and R. G. Wenzel, J. Chem. Phys. 49, 4365 (1968).

${ }^{19}$ R. Ross, P. Andersson, and G. Bäckström, High Temp.-High Pressures 9, 87 (1977).

${ }^{20} \mathrm{G}$. Honjo, N. Kitamura, K. Shimaoka, and K. Mihama, J. Phys. Soc. Jpn. 11, 527 (1956).

${ }^{21}$ L. G. Dowell and A. P. Rinfret, Nature (London) 188, 1144 (1960).

${ }^{22}$ O. Mishima, L. D. Calvert, and E. Whalley, Nature 314, 76 (1985).

${ }^{23}$ G. P. Johari, A. Hallbrucker, and E. Mayer, J. Phys. Chem. 94, 1212 (1990).

${ }^{24}$ A. Elarby-Aouizerat, J.-F. Jal, J. Dupuy, H. Schildberg, and P. Chieux, J. Phys. Colloq. 48(C1), 465 (1987).

${ }^{25}$ G. Vigier, G. Thollet, and R. Vassoile, J. Cryst. Growth 84, 309 (1987).

${ }^{26}$ G. P. Johari, J. Phys. Chem. B 102, 4711 (1998).

${ }^{27}$ G. P. Johari, J. Chem. Phys. 122, 194504 (2005).

${ }^{28}$ T. L. Malkin, B. J. Murray, A. V. Brukhno, J. Anwar, and C. G. Salzmann, Proc. Natl. Acad. Sci. U. S. A. 109, 1041 (2012).

${ }^{29}$ S. Takeya, T. Uchida, J. Nagao, R. Ohmura, W. Shimada, Y. Kamata, T. Ebunuma, and H. Narita, Chem. Eng. Sci. 60, 1383 (2005).

${ }^{30}$ A. Falenty and W. F. Kuhs, J. Phys. Chem. B 113, 15975 (2009).

${ }^{31}$ W. F. Kuhs, C. Sippel, A. Falenty, and T. C. Hansen, Proc. Natl. Acad. Sci. U. S. A. 109, 21259 (2012).

${ }^{32}$ C. G. Salzmann, E. Mayer, and A. Hallbrucker, Phys. Chem. Chem. Phys. 6, 1269 (2004).

${ }^{33}$ D. D. Klug, Y. P. Handa, J. S. Tse, and E. Whalley, J. Chem. Phys. 90, 2390 (1989).

${ }^{34}$ C. A. Tulk and D. D. Klug, Phys. Rev. B 63, 212201 (2001), showed that ice XII at 1 bar amorphizes on annealing and then transforms on further annealing to ice Ic; Later, C. G. Salzmann, I. Kohl, T. Loerting, E. Mayer, and A. Hallbrucker, Phys. Chem. Chem. Phys. 5, 3507 (2003), confirmed the transformation of ice XII to ice Ic by using DSC heating scans at $30 \mathrm{~K} / \mathrm{min}$.

${ }^{35}$ W. F. Kuhs, G. Genov, D. K. Staykova, and T. Hansen, Phys. Chem. Chem. Phys. 6, 4917 (2004); See also, A. Falenty, T. C. Hansen, and W. F. Kuhs, in Physics and Chemistry of Ice 2010, edited by Y. Furukaawa, G. Sazaki, T. Uchida, and N. Watanabe (Hakkaido University Press, Sapporo, 2011), p. 411.

${ }^{36}$ T. L. Malkin, B. J. Murray, C. G. Salzmann, V. Molinero, S. J. Pickering, and T. F. Whale, Phys. Chem. Chem. Phys. 17, 60 (2015).

${ }^{37}$ W. F. Kuhs, D. V. Bliss, and J. L. Finney, J. Phys. Colloq. 48, 631 (1987). Three features in the neutron powder diffraction led to the interpretation that ice Ic contains stacking faults: (i) the 111 peak is broadened, (ii) a small peak appears at the position where the $10 \overline{1} 0$ reflection of ice Ih is found, and (iii) the relative intensities of the peaks differ from those calculated for ice $\mathrm{Ih}$ and ice Ic. On the basis of previous knowledge on the formation of stacking faults by glide dislocations, Petrenko and Whitworth [Physics of Ice (Oxford University Press, 1999)] described formation of stacking faults in ice Ih before considering those in ice Ic. For further details, we refer readers to their monograph. Shallcross and Carpenter [J. Chem. Phys. 26, 782 (1957).] had found evidence of hexagonal ice peak in the X-ray diffraction data of their cubic ice made by vapor deposition. But Bartel-Rausch et al. [Rev. Mod. Phys. 84, 885 (2012).] wrote: "After the first identification of ice Ic (König, 1943) it was realized by Shallcross and Carpenter (1957) that the diffraction pattern of ice Ic had hexagonal components, wrongly interpreted by them as an admixture of ice Ih.” In Sec. B of their paper, Bartel-Rausch et al. succinctly reviewed the previous studies by Kuhs and coworkers.

${ }^{38} \mathrm{D}$. Eisenberg and W. Kauzmann, The Structure and Properties of Water (Oxford University Press, 1969).

${ }^{39}$ P. V. Hobbs, Ice Physics (Clarendon, Oxford, 1974).

${ }^{40}$ V. F. Petrenko and R. W. Whitworth, Physics of Ice (Oxford University Press, 1999).

${ }^{41}$ L. E. Murr, Interfacial Phenomena in Metals and Alloys (Addison-Wesley Pub. Co., 1975).

${ }^{42} \mathrm{P}$. Haasen, Physical Metallurgy, 2nd ed. (Cambridge University Press, NY, 1985), translated by J. Mordike.

${ }^{43}$ M. S. Paterson, J. Appl. Phys. 23, 805 (1952).

${ }^{44}$ T. Hondoh, T. Itoh, S. Amakai, K. Goto, and A. Higashi, J. Phys. Chem. 87, 4040 (1983).

${ }^{45}$ M. Oguro and T. Hondoh, "Stacking faults in ice crystals," in Lattice Defects in Ice Crystals, edited by A. Higashi (Hakkaido University Press, Sapporo, 1988), pp. 49-67. They reported the stacking fault energy in the range, 0.1$0.3 \mathrm{~mJ} \mathrm{~m}^{-2}$. 
${ }^{46}$ E. B. Moore and V. Molinero, Phys. Chem. Chem. Phys. 13, 20008 (2011).

${ }^{47}$ G. Bullock and V. Molinero, Faraday Discuss. 167, 371 (2013).

${ }^{48}$ A. Reinhardt, J. P. K. Doye, E. G. Noya, and C. Vega, J. Chem. Phys. 137, 194504 (2012).

${ }^{49}$ P. Pirzadeh and P. G. Kusalik, J. Am. Chem. Soc. 133, 704 (2011).

${ }^{50}$ L. Bosio, G. P. Johari, and J. Teixeira, Phys. Rev. Lett. 56, 460 (1986).

${ }^{51}$ M. Riikonen, M. Sillanpä, L. Virta, D. Sullivan, J. Moilanen, and I. Luukkonen, Appl. Opt. 39, 6080 (2000).

${ }^{52}$ H. Kiefte, M. J. Clouter, and E. Whalley, J. Chem. Phys. 81, 1419 (1984).

${ }^{53}$ T. Takahashi, J. Cryst. Growth 59, 441 (1982).

${ }^{54}$ C. W. Lee [J. Meteorol. Soc. Jpn. 50, 171 (1972)] and Uyeda and Kikuchi [J. Fac. Sci., Univ. Tokyo, Sect. 1A 5, 21 (1976)] had found that the $c$ axes of the crystallites in a dendritic snow flake are oriented at an angle of $70^{\circ}$ to one another. These data have been analyzed by T. Kobayashi and co-workers [J. Cryst. Growth 35, 262 (1976) and J. Cryst. Growth 64, 593 (1983).], who concluded that the ice nuclei that first form on freezing of water droplets in the atmosphere are octahedral crystals of cubic ice. Further vapor condensation produces hexagonal ice by epitaxial growth on the (111) planes of cubic ice nuclei. T. Takahashi [J. Cryst. Growth 59, 441 (1982), Ref. 53, above] has shown that the junction of the twins crystals has a low energy. Taken from Ref. 27 here.

${ }^{55}$ J. Goodman, O. B. Toon, R. F. Pueschel, K. G. Snetsinger, and S. Verma, J. Geophys. Res.: Atmos. 94, 16449, doi:10.1029/JD094iD14p16449 (1989).

${ }^{56}$ L. F. Keyser and M. T. Leu, Microsc. Res. Tech. 25, 434 (1993).

${ }^{57}$ T. Hansen, M. M. Koza, and W. F. Kuhs, J. Phys.: Condens. Matter 20, 285104 (2008).

${ }^{58}$ J. E. Bertie and M. Jacobs, J. Chem. Phys. 67, 2445 (1977).

${ }^{59}$ T. H. G. Carr, J. J. Shephard, and C. G. Salzmann, J. Phys. Chem. Lett. 5, 2469 (2014).

${ }^{60}$ Y. P. Handa, D. D. Klug, and E. Whalley, J. Chem. Phys. 84, 7009 (1986).

${ }^{61}$ A. Hallbrucker and E. Mayer, J. Phys. Chem. 91, 503 (1987).

${ }^{62}$ O. Yamamuro, M. Oguni, T. Matsuo, and H. Suga, J. Phys. Chem. Solids 48, 935 (1987).

${ }^{63}$ H. Tanaka and I. Okabe, Chem. Phys. Lett. 259, 593 (1996).

${ }^{64}$ J. E. Shilling, M. A. Tolbert, O. B. Toon, E. J. Jensen, and B. J. Murray, Geophys. Res. Lett. 33, L17801, doi:10.1029/2006GL026671 (2006).

${ }^{65}$ R. Chidambaram, Acta Crystallogr. 14, 467 (1961).

${ }^{66} \mathrm{E}$. Whalley, "The hydrogen bond in ice," in The Hydrogen Bond-Recent Developments in Theory and Experiments, III. Dynamics, Thermodynamics and Special Systems, edited by P. Schuster, G. Zundel, and C. Sandorfy (North Holland, New York, 1976), Chap. 29, pp. 1425-1470.

${ }^{67}$ O. Andersson and A. Inaba, Phys. Chem. Chem. Phys. 7, 1441 (2005).
${ }^{68}$ O. Andersson and H. Suga, Phys. Rev. B 50, 6583 (1994).

${ }^{69}$ G. P. Johari and O. Andersson, J. Chem. Phys. 120, 6207 (2004).

${ }^{70}$ O. Andersson and H. Suga, Phys. Rev. B 65, 140201 (2001).

${ }^{71}$ T. C. Hansen, C. Sippel, and W. F. Kuhs, Z. Kristallogr. 230, 75 (2015)

${ }^{72}$ T. C. Hansen, M. M. Koza, P. Lindner, and W. F. Kuhs, J. Phys.: Condens. Matter 20, 285105 (2008).

${ }^{73}$ N. W. Ashcroft and N. D. Mermin, Solid State Physics (Saunders College Publishing, Philadelphia, 1976), pp. 258-501.

${ }^{74}$ G. P. Johari, W. Pascheto, and S. J. Jones, J. Chem. Phys. 100, 4548 (1994).

${ }^{75}$ G. Salvetti, E. Tombari, and G. P. Johari, J. Chem. Phys. 102, 4987 (1995).

${ }^{76}$ G. P. Johari, J. Chem. Phys. 109, 1070 (1998).

${ }^{77}$ J. W. Gibbs, The Collected Works. Vol. 1. Thermodynamics (Longman, Greens, NY, 1928).

${ }^{78}$ R. Defay, I. Prigogine, and A. Bellemans, Surface Tension and Adsorption (Longmans, London, 1966), p. 22 translated by D. H. Everett.

${ }^{79}$ G. P. Johari, Philos. Mag. A 77, 1367 (1998). This paper describes thermodynamic contributions from pre-melting or pre-transformation of finely dispersed crystals.

${ }^{80}$ G. P. Johari, Philos. Mag. B 78, 375 (1998). It was argued that when ice Ih and Ic are joined along their close-packed planes, with the close-packed directions parallel, the resulting interface is completely coherent and the interfacial energy is low. No other two planes can form a coherent interface because no other planes are identical in both crystal lattices. Because of the interfacial or strain energy at the interface, the growth of ice Ih in ice Ic is determined by diffusion of defects in the vicinity of the interface. In Ref. 31 above, Kuhs et al. noted that the arguments based on grain boundaries and strain energies between crystallites used to explain the extended range of ice Ic to ice Ih transformation would not be valid for loosely connected aggregates of their vapor deposited ice Ic.

${ }^{81}$ I. Kohl, E. Mayer, and A. Hallbrucker, Phys. Chem. Chem. Phys. 2, 1579 (2000).

${ }^{82}$ B. J. Murray, C. G. Salzmann, A. J. Heymsfield, S. Dobbie, R. R. Neely III, and C. J. Cox, "Trigonal ice crystals in Earth's atmosphere," Bull. Am. Meteorol. Soc. (published online 2014).

${ }^{83}$ A. Guinier, G. B. Bokij, K. Boll-Dornberger, J. M. Cowley, S. Durovic, H. Jagodzinski, P. Krishna, P. M. de Wolff, B. B. Zvyagin, D. E. Cox, P. Goodman, Th. Hahn, K. Kuchitsu, and S. C. Abrahams, Acta Crystallogr., Sect. A: Found. Crystallogr. 40, 399 (1984).

${ }^{84}$ G. P. Johari, Phys. Chem. Chem. Phys. 2, 1567 (2000).

${ }^{85}$ H. Suga and S. Seki, Faraday Discuss. Chem. Soc. 69, 221 (1980).

${ }^{86}$ P. Németh, L. A. J. Garvie, T. Aoki, N. Dubrovinskaia, L. Dubrovinsky, and P. R. Buseck, Nat. Commun. 5, 5447 (2014). See also references therein.

${ }^{87}$ W. G. Bergers, Physica 1, 561 (1934). 